\title{
Incremento de la contaminación por microplásticos en aguas superficiales de la bahía de Buenaventura, Pacífico colombiano
}

\section{Increase in microplastic pollution in the surface waters of Buenaventura Bay, Colombian Pacific}

\author{
Laura Vidal ${ }^{1}$,Andrés Molina ${ }^{l}$ y Guillermo Duque ${ }^{2} *$ \\ (iD) $0000-0002-1033-4317$ (iD) $0000-0003-1954-4393$ (iD) $0000-0002-2468-529 X$ \\ 1. Grupo de Investigación Ecología y Contaminación Acuática, Universidad Nacional de Colombia, Sede Palmira, Carrera 32 Chapinero- vía \\ Candelaria,Palmira,Colombia.lvvidalt@unal.edu.co,aemolinas@unal.edu.co \\ 2. Facultad de Ingeniería y Administración, Universidad Nacional de Colombia, Sede Palmira, Carrera 32 Chapinero- vía Candelaria, Palmira, \\ Colombia.gduquen@unal.edu.co* \\ * Autor de correspondencia.
}

\section{RESUMEN}

$\mathrm{L}$ a contaminación por microplásticos (MP) en los ecosistemas costeros es un grave problema alrededor del mundo. En Colombia existe poca información sobre este contaminante, especialmente para la costa pacífica. Por lo anterior, se determinó la densidad, distribución y tipos de MP en la superficie del agua del estuario de la bahía de Buenaventura, principal puerto de Colombia. Se recolectaron 36 muestras en dos sectores: Estuario Interno (EI), más cercano a la principal zona urbana y la desembocadura de ríos y el Estuario Externo (EE), más alejado de ambas influencias; el trabajo se llevó a cabo durante tres épocas: 1luvia, transición y seca, en 2015 y 2019. En promedio, la densidad de MP aumentó anualmente un 28,7\% en las aguas superficiales de la bahía de Buenaventura. La densidad de los MP aumentó en ambos años al incrementar la distancia desde la zona interna de la bahía, observándose una menor densidad en el EI (271 928,6 \pm 60 734,9 partículas $\left./ \mathrm{km}^{2}\right)$ y una mayor densidad en el EE (492 106,2 $\pm 98467,1$ partículas $\left./ \mathrm{km}^{2}\right)$. Así mismo, se encontraron las mayores densidades de MP en las épocas de lluvia $\left(664766,7 \pm 129828,1\right.$ partículas $\left./ \mathrm{km}^{2}\right)$ y transición $\left(320386,5 \pm 59313,7\right.$ partículas $\left.\mathrm{km}^{2}\right)$, mientras que en la época seca se presentaron en menores concentraciones (160 $899 \pm 43606$ partículas $/ \mathrm{km}^{2}$ ), lo cual se asocia al incremento de la escorrentía en épocas con mayor precipitación. Los MP hallados se clasificaron según su forma en fragmentos, fibras y pellets. En el EI las fibras se presentaron en mayor cantidad, mientras en que en el EE predominaron los fragmentos. Los tipos de MP que más abundaron en la superficie fueron los fragmentos $(62,2 \%)$ y las fibras $(37,4 \%)$, posiblemente por su poca densidad en comparación a la densidad del agua estuarina.

PALABRAS CLAVE: Bahía de Buenaventura, microplástico, variación espacial, épocas climáticas, monitoreo.

\section{ABSTRACT}

$\mathrm{M}$ icroplastic (MP) pollution in coastal ecosystems is a serious problem throughout the world. In Colombia, there is little information on this issue, especially for the Pacific coast. For these reasons, the density, distribution, and types of MP on the surface of the water were studied in the bay of Buenaventura estuary, Colombia's main seaport. A total of 36 samples were collected in two estuarine areas: the inner estuary (IE), closest to the main urban area and river mouths, and the external estuary (EE), further away from both areas, during three seasons: rainy, transition, and dry, in 2015 and 2019. On average, the density of the MP increases annually by $28.7 \%$ in the surface waters of Buenaventura Bay. It was also found that the highest densities of MP occurred mainly in the rainy season $\left(664,766.7 \pm 129,828.1 \mathrm{particles} / \mathrm{km}^{2}\right)$ and transition $\left(320,386.5 \pm 59,313.7\right.$ particles $\left./ \mathrm{km}^{2}\right)$, while in the dry season the densities were lower $\left(160,899 \pm 43,606 \mathrm{particles} / \mathrm{km}^{2}\right)$, due to increased runoff in times of higher rainfall. In both years, MP concentration increased the further from the inner zone of the bay the sample was taken, with a lower density observed in the IE $\left(271,928.6 \pm 60,734.9 \mathrm{particles} / \mathrm{km}^{2}\right)$ and a higher density in the EE $\left(492,106.2 \pm 98,467.1 \mathrm{particles} / \mathrm{km}^{2}\right)$. The MP found were classified into fragments, fibers, and pellets, according to their shape. In the IE, there was a greater quantity of fibers, while in the EE, fragments where the most common MP shape. The types of MP that were more abundant on the surface were fragments $(62.2 \%)$ and fibers (37.4\%), possibly due to their low density compared to the density of the estuarine water.

KEYWORDS: Buenaventura Bay, microplastics, spatial variation, climate seasons, monitoring. 


\section{INTRODUCCIÓN}

Los plásticos son productos que se generan en grandes cantidades, cerca de 400 millones de toneladas de plástico se producen anualmente alrededor del mundo (Devasahayam et al., 2019). En su mayoría, son dispuestos de manera inadecuada, por falta de eficientes sistemas de gestión de residuos sólidos y una inadecuada planificación de los asentamientos urbanos e industriales (Das et al., 2019; Vanapalli et al., 2019). Su acumulación está generando graves problemas ambientales ya que estos productos llegan a distintos ecosistemas acuáticos y terrestres, afectando las diversas formas de vida que en ellos habitan (Caruso, 2019). Los plásticos presentan una degradación lenta, la cual se produce a partir de la acción mecánica, biodegradación y fotooxidación (Ma et al., 2019). A pesar de que la probabilidad de biodegradación de estos productos sea muy baja, su descomposición se refleja en los cambios de las características mecánicas, ópticas o eléctricas, lo cual genera grietas, erosión, decoloración y la fragmentación de los mismos (Singh y Sharma, 2008). Debido a la fragmentación de los plásticos, se originan objetos de menor tamaño. Aquellos que poseen un tamaño inferior a $5 \mathrm{~mm}$ de diámetro se conocen como micro plásticos (MP) (Alimba y Faggio, 2019). Los MP se clasifican como primarios y secundarios. Los primarios son aquellos que están diseñados y fabricados con éstos tamaños para un fin en específico, por ejemplo, se encuentran usualmente presentes en limpiadores faciales y corporales (exfoliantes), cosméticos y microfibras sintéticas de ropa (Pan et al., 2019). Los secundarios hacen referencia a aquellos que se originan a partir de la fragmentación de objetos plásticos de un tamaño superior (Cole et al., 2011).

Actualmente, se estima que alrededor de 245 millones de toneladas anuales de MP ingresan al medio marino a través del turismo, aguas residuales, pesca y/o derrames industriales (Alimba y Faggio, 2019). Se han encontrado MP en los ecosistemas marinos alrededor del mundo con diferentes características y en concentraciones considerables (Lima et al., 2016; Invemar y MADS, 2017; Schmidt et al., 2018; Nie et al., 2019; Kor y Mehdinia, 2020). Su abundancia en el mar ha traído consigo importantes afectaciones negativas en los organismos marinos y en los procesos ecológicos, debido a que su existencia en las superficies oceánicas es ubicua (Pan et al., 2019). Por su pequeño tamaño, los MP se encuentran disponibles para la ingestión de muchos organismos vivos de diferentes niveles tróficos, provocando efectos nocivos, ya que la mayoría de los organismos marinos no metabolizan los MP a causa de la inexistencia de una enzima especializada en

\section{INTRODUCTION}

Plastics are produced in huge quantities, with about 400 million tons of plastic produced annually across the world (Devasahayam et al., 2019). The majority is disposed of improperly, due to a lack of efficient solid waste management systems and inadequate planning of urban and industrial settlements (Das et al., 2019; Vanapalli et al., 2019). The accumulation of plastics in different aquatic and terrestrial ecosystems is causing serious environmental problems and affecting the various forms of life that inhabit them (Caruso, 2019). Plastics degrade slowly through mechanical action, biodegradation and photo-oxidation (Ma et al., 2019). The likelihood that these products will biodegrade is very low, and so when they decompose, their mechanical, optical, or electrical characteristics start to change, leading to cracking, erosion, discoloration and fragmentation (Singh and Sharma, 2008). Smaller objects are created as plastics start to fragment. Pieces smaller than 5 $\mathrm{mm}$ in diameter are termed microplastics (MP) (Alimba and Faggio, 2019), and can be classified as primary or secondary MP. Primary MP are designed and manufactured at these sizes for a specific purpose, e.g., those usually found in facial and body cleansers (exfoliants), cosmetics and synthetic microfibers in clothing (Pan et al., 2019). Secondary MP are those that originate from the fragmentation of larger plastic objects (Cole et al., 2011).

Currently, it is estimated that around 245 million tons of MP enter the marine environment annually via tourism, sewage, fisheries and/or industrial spills (Alimba and Faggio, 2019). Considerable concentrations of MP with different characteristics have been found in marine ecosystems around the world (Lima et al., 2016; Invemar and MADS, 2017; Schmidt et al., 2018; Nie et al., 2019; Kor and Mehdinia, 2020). The abundance of MP in the sea has resulted in significant negative impacts on marine organisms and ecological processes, due to their pervasive presence on ocean surfaces (Pan et al., 2019). Due to their small size, MP can be ingested by many living organisms at different trophic levels and cause them harm, as most marine organisms do not metabolize MP, lacking an enzyme that specializes in the decomposition of synthetic polymers (microplastics) (Guzzetti et al., 2018).

In vertebrate organisms, the ingestion of plastic debris causes obstruction of the intestines, resulting in intestinal lesions, morbidity, and mortality. It has also been found that these elements lead to mechanical obstruction of 
la descomposición de polímeros sintéticos (microplásticos) dentro de sus organismos (Guzzetti et al., 2018).

En organismos vertebrados la ingestión de desechos plásticos genera la obstrucción de los intestinos, provocando lesiones intestinales, morbilidad y mortalidad. Asimismo, se ha encontrado que estos elementos generan obstrucciones mecánicas de movimiento, atrapamiento, ahogamiento, restringe la alimentación y produce daños en la piel y el sistema esquelético de estos organismos (Alimba y Faggio, 2019). Los MP, por su parte, después de ser ingeridos y acumulados dentro del organismo pueden movilizarse entre tejidos o también ser eliminados a través de la excreción. La acumulación de este material puede generar lesiones al interior o exterior del organismo, bloqueos intestinales, alteraciones alimenticias, problemas de fertilidad y disminución de la supervivencia de las crías (Guzzetti et al., 2018). Por otro lado, los MP juegan un papel importante como vectores de contaminantes biológicos y químicos dentro de las cadenas tróficas (Carbery et al., 2019; Caruso, 2019). Se han encontrado MP en organismos pequeños como zooplancton (Botterell et al., 2019), mejillones (Giacomo et al., 2015), pepinos de mar (Mohsen et al., 2019), entre otros; así como en organismos muchos más grandes tales como peces (Chan et al., 2019), aves (alimentadas con organismos contaminados) (Provencher et al., 2018), tortugas (Caron et al., 2018), cetáceos (Zhu et al., 2019) y otros mamíferos marinos.

En el medio marino se lleva a cabo la mitad de la producción primaria del planeta, la cual proporciona energía necesaria para albergar una importante biodiversidad (Sarma y Delabehera, 2019). Así mismo, los ecosistemas marinos funcionan como importantes sumideros de carbono y son la base del sustento económico de muchos países a través del turismo y de la producción pesquera. Sin embargo, cada día las actividades antrópicas, incluyendo la contaminación por MP, deterioran estos ecosistemas, impidiendo que se lleven a cabo de forma eficiente los procesos naturales y se vea afectada la vida silvestre, el sistema ecológico y la salud humana a través de las vías de alimentación (Alimba y Faggio, 2019). A causa de este constante deterioro, se han desarrollado distintas investigaciones con el objetivo de identificar los sitios prominentes por donde ingresan los MP al medio marino y han encontrado que uno de los mayores aportes se realiza a partir de los estuarios (Zhao et al., 2019), los cuales son áreas importantes debido a la diversidad de especies que alberga, a su producción biológica y como fuente o sumidero de distintos materiales de los ecosistemas costeros (Invemar, 2017a). Por otro lado, brindan una gran variedad de servicios movement, entrapment, drowning, restricted feeding and damage to the skin and skeletal system of these organisms (Alimba and Faggio, 2019). Furthermore, after being ingested and having started to accumulate inside the organism, MP can move between tissues or also be eliminated through excretion. The accumulation of this material can cause lesions to the inside or outside of the organism, intestinal blockages, changes in feeding, fertility problems and decreased survival of offspring (Guzzetti et al., 2018). MP also play an important role as vectors of biological and chemical contaminants within food webs (Carbery et al., 2019; Caruso, 2019). They have been found in small organisms such as zooplankton (Botterell et al., 2019), mussels (Giacomo et al., 2015), and sea cucumbers (Mohsen et al., 2019) among others; as well as in much larger organisms such as fish (Chan et al., 2019), birds (having fed on contaminated organisms) (Provencher et al., 2018), turtles (Caron et al., 2018), cetaceans (Zhu et al., 2019) and other marine mammals.

Half of the planet's primary production takes place in the marine environment, and provides the energy needed to support important biodiversity (Sarma and Delabehera, 2019). Marine ecosystems also function as important carbon sinks and are the basis of the economic livelihood of many countries through tourism and fisheries production. However, these ecosystems are being deteriorated every day by anthropogenic activities including MP pollution, which prevent natural processes from occurring efficiently and affect wildlife, the ecological system and human health by means of food pathways (Alimba and Faggio, 2019). In light of this constant deterioration, research has been carried out to identify the main sites through which MP enter the marine environment, finding estuaries to be one of the major sources (Zhao et al., 2019). These are important environments as hosts of a diversity of species, their biological production, and their role as sources or sinks of different materials in coastal ecosystems (Invemar, 2017a). On the other hand, they provide a wide variety of ecosystem services, such as recreation, tourism, nutrient recycling, food and raw material production, carbon sequestration, and coastal protection from extreme weather events (García et al., 2018).

Buenaventura Bay is an estuary, through which the Dagua, Caimán, Limones, Anchicayá and Raposo rivers drain into the sea, home to communities of benthos, macroinvertebrates and fish that are vulnerable to diverse types of pollution (Gamboa et al., 2018; Martínez et al., 2019; Duque et al., 2020; Molina et al., 2020), including 
ecosistémicos, tales como recreación, turismo, reciclaje de nutrientes, producción de alimento y materia prima, captación de carbono y protección de las costas ante eventos climáticos extremos (García et al., 2018).

La bahía de Buenaventura es un estuario, formado por la desembocadura de los ríos Dagua, Caimán, Limones, Anchicayá y Raposo, que alberga comunidades de bentos, macroinvertebrados y peces vulnerables a diferentes tipos de contaminación (Gamboa et al., 2018; Martínez et al., 2019; Duque et al., 2020; Molina et al., 2020), incluyendo los microplásticos (Tafurt, 2020; Vásquez et al., 2021). En Buenaventura, la calidad de las redes hídricas ha sido deteriorada por el vertimiento de aguas residuales domésticas. Otras fuentes de contaminación son los residuos de minería, las escorrentías agrícolas y ganaderas, así como los residuos de la industria pesquera y del puerto de Buenaventura. Como consecuencia se ha afectado la calidad ambiental de este ecosistema estuarino (Troncoso et al., 2009; Invemar, 2018). Además de la carga contaminante que llega a partir del medio fluvial también muchos sectores del estuario han sido usados como sitios de descarga de sustancias contaminantes tales como sólidos domésticos e industriales, MP y derrames de derivados del petróleo (Invemar, 2005; Invemar, 2018). Los estuarios son un foco de interés para el estudio de MP dado a que funcionan como un medio de ingreso de este material contaminante a los medios marinos desde las aguas continentales (Zhang et al., 2019). Debido a lo anterior, el propósito de este estudio fue realizar una determinación espaciotemporal de la densidad, distribución y tipos de MP en la superficie del agua de la bahía de Buenaventura, Pacífico colombiano.

\section{ÁREA DE ESTUDIO}

La presente investigación se realizó en la bahía de Buenaventura, la cual se encuentra ubicada entre las latitudes $3^{\circ} 44^{\prime}-56^{\prime} \mathrm{N}$ y las longitudes $77^{\circ} 01^{\prime}-20^{\prime}$ (Figura 1). Posee un ancho aproximado de 3,4 km en la entrada del mar y 5,5 km en la parte interna. Su extensión es de $30 \mathrm{~km}$ lo que le confiere una forma estrecha y alargada (Otero, 2005) y su profundidad promedio es $5 \mathrm{~m}$. Las épocas hidroclimáticas en la bahía de Buenaventura se pueden clasificar como bimodales, con mayores precipitaciones de septiembre a octubre, y menores entre febrero y marzo (Otero et al., 2007; Guzmán et al., 2014). Adicionalmente, tiene una ubicación próxima a la Cordillera Occidental del territorio colombiano, lo que genera un enfriamiento de las corrientes de aire cálido y húmedo, causando intensas precipitaciones (Cantera y Blanco, 2001). microplastics (Tafurt et al., 2021; Vásquez et al., 2021). In Buenaventura, the discharge of domestic wastewater has lowered the water quality of river networks. Other sources of contamination include mining waste, agricultural and livestock runoff, as well as waste from the fishing industry and the port of Buenaventura. This has affected the environmental quality of this estuarine ecosystem (Invemar, 2018; Troncoso et al., 2009). In addition to the pollution brought downriver, many zones of the estuary have also been used to dump pollutants such as domestic and industrial solids, MP, and oil byproducts (Invemar, 2005; Invemar, 2018). Estuaries are of interest for the study of MP since they transport this pollutant into the marine environment from inland waters (Zhang et al., 2019). For all these reasons, this study sought to carry out a spatiotemporal determination of the density, distribution and types of MP in the surface waters of Buenaventura Bay in the Colombian Pacific.

\section{STUDY AREA}

This research was conducted in Buenaventura Bay, situated between latitudes $3^{\circ} 44^{\prime}-56^{\prime} \mathrm{N}$ and longitudes $77^{\circ}$ $01^{\prime}-20$ ' (Figure 1). It is approximately $3.4 \mathrm{~km}$ wide at the sea mouth and the inner bay is $5.5 \mathrm{~km}$ across. It is $30 \mathrm{~km} \mathrm{long}$, which gives it a narrow and elongated shape (Otero, 2005), and its average depth is $5 \mathrm{~m}$. The hydroclimatic seasons in Buenaventura Bay can be classified as bimodal, with higher rainfall from September to October, and lower rainfall between February and March (Otero et al., 2007; Guzmán et al., 2014). It is also positioned close to Colombia's Cordillera Occidental mountain range, which cools the warm and humid air currents, causing intense precipitation (Cantera and Blanco, 2001). The average annual sea surface temperature is $28.2{ }^{\circ} \mathrm{C}$, with average monthly minimum temperatures of $27.8^{\circ} \mathrm{C}$ in November and maximums of $28.5^{\circ} \mathrm{C}$ in March (Otero, 2005). The bay is made up of two large, well-defined sectors: the external bay and the inner bay (Invemar and CVC, 2015). The external bay has direct communication with the open sea, and is therefore strongly influenced by it. The inner bay is considered a well-mixed estuary and is greatly affected by freshwater from the rivers and estuaries that flow into it (Invemar and CVC, 2015). 


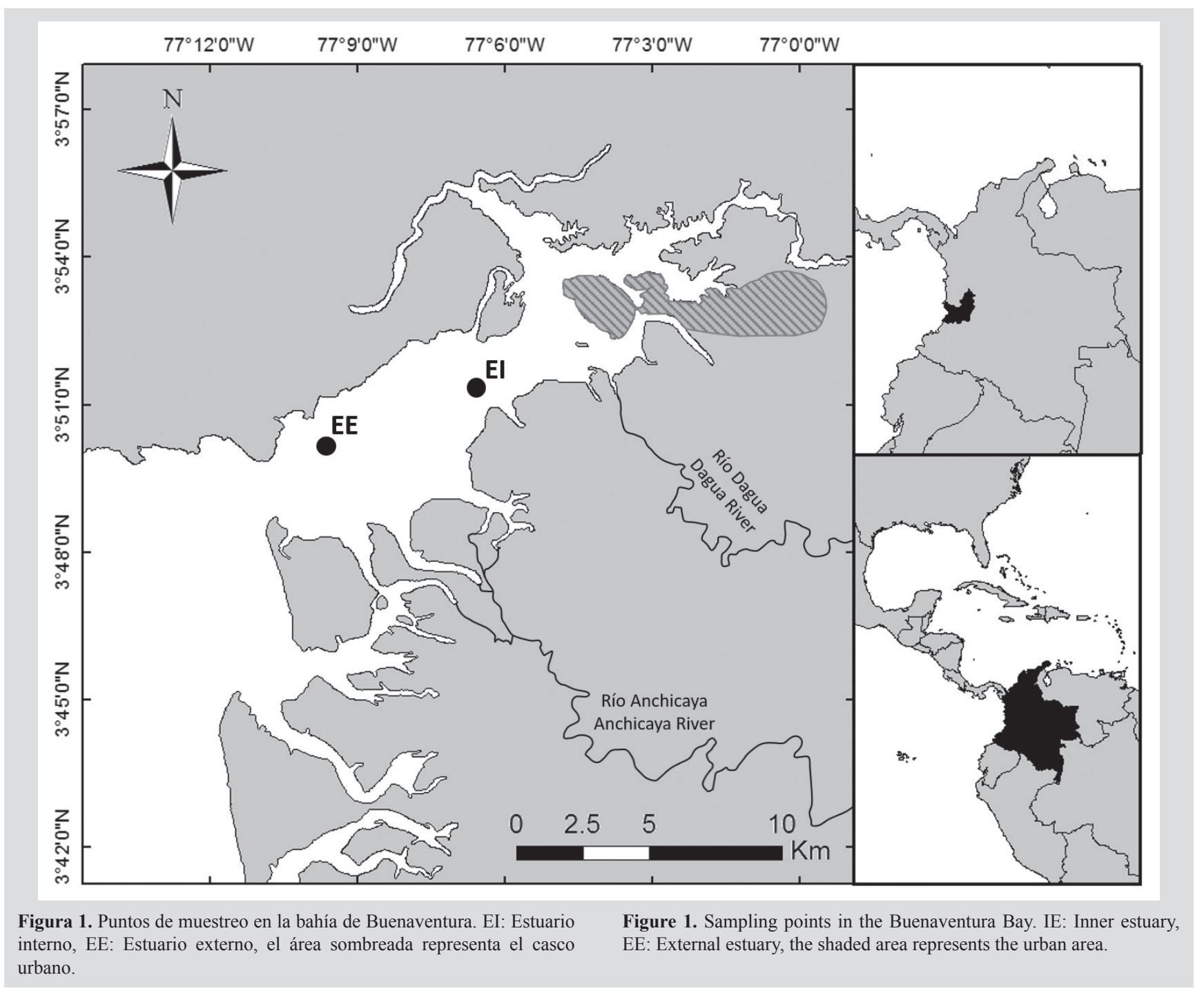

La temperatura promedio anual de la superficie del mar es de $28,2{ }^{\circ} \mathrm{C}$, con valores promedios mensuales mínimos de $27,8^{\circ} \mathrm{C}$ en noviembre y máximos de $28,5^{\circ} \mathrm{C}$ en marzo (Otero, 2005). Esta bahía está conformada por dos grandes sectores bien definidos: la bahía exterior y la bahía interior (Invemar y CVC, 2015). La bahía exterior tiene comunicación directa con el mar abierto, por lo que se ve fuertemente influenciada por el mismo. La bahía interior se considera como un estuario bien mezclado y tiene una fuerte influencia del agua dulce de los ríos y esteros que en ella desembocan (Invemar y CVC, 2015).

\section{METODOLOGÍA}

\section{Fase de Campo}

Para este estudio se colectó agua superficial en seis muestreos realizados en los meses de abril, julio y noviembre de 2015, y marzo, junio y octubre de 2019 ,

\section{METHODOLOGY}

Fieldwork phase

For this study, six surface water samples were collected during the months of April, July and November 2015, and March, June and October 2019, corresponding to the region's predominant climatic periods: dry season (March-April), transition season (June-July) and rainy season (October-November). In each season, three replicates were taken in two zones of the estuary, one from the inner estuary (IE) and another from the external estuary (EE). Station IE $\left(77^{\circ} 6^{\prime} 33.1^{\prime \prime} \mathrm{W}\right.$ and $\left.3^{\circ} 50^{\prime} 51.5^{\prime \prime} \mathrm{N}\right)$ is an area affected by the mouth of the river Dagua and is closer to the urban center and possible sources of contamination. Station $\mathrm{EE}\left(77^{\circ} 9^{\prime} 35.9^{\prime \prime} \mathrm{W}\right.$ and $\left.3^{\circ} 50^{\prime} 58.7^{\prime \prime} \mathrm{N}\right)$ is farther away from the main urban area and is more affected by the sea (Figure 1). The spatial distribution of the sampling points follows 
correspondientes a los periodos climáticos predominantes en la región: época seca (marzo-abril), época de transición (junio-julio) y época de lluvia (octubre-noviembre). En cada época, se tomaron tres réplicas en dos zonas del estuario, una estación en el estuario interno (EI) y otra en el estuario externo (EE). La estación EI ( $77^{\circ} 6^{\prime} 33,1^{\prime}$ ” W y $3^{\circ} 50^{\prime} 51,5^{\prime} \mathrm{N}$ ) es un área influenciada por la desembocadura del río Dagua y más cercana al casco urbano y a posibles fuentes de contaminación. La estación EE (77 9' 35,9” W y $3^{\circ} 50^{\prime} 58,7^{\prime}$ N) se encuentra más alejada de la principal zona urbana y presenta mayor influencia marina (Figura 1). La distribución espacial de los puntos de muestreo representa un posible gradiente de concentración de MP que se encuentra en la bahía. También, los muestreos en las épocas secas, transición y lluvia, permiten evaluar el efecto de los cambios de la precipitación y la escorrentía sobre la concentración de los MP.

En cada punto (EI y EE) y época del año (seca, transición y lluvia) se recolectaron muestras de agua superficial, mediante una red de arrastre horizontal de 250 micras, con un diámetro de abertura de $29,5 \mathrm{~cm}$. Los arrastres se hicieron en una distancia de 600,4 $\pm 50,7 \mathrm{~m}$ (promedio $\pm \mathrm{DE}$ ), con una duración de 10 minutos, a una velocidad constante. Cada una de las muestras recolectadas tuvo tres réplicas para un total de 36 muestras. Estas muestras se transfirieron a recipientes limpios y posteriormente se refrigeraron a una temperatura de $4{ }^{\circ} \mathrm{C}$.

Fase de laboratorio

Para el tratamiento de las muestras se adaptaron las metodologías para el análisis de MP propuestas por Masura et al. (2015) e Invemar (2017a). Inicialmente se hizo un proceso de separación, donde cada muestra recolectada se transfirió con ayuda de agua destilada a una disposición apilada de tamices en acero inoxidable de $5 \mathrm{~mm}$ y $0,25 \mathrm{~mm}$ de diámetro. Los elementos retenidos en el tamiz de $5 \mathrm{~mm}$ y aquellos que pasaron por el tamiz de $0,25 \mathrm{~mm}$ fueron desechados, ya que no corresponden al tamaño del material en estudio. Los sólidos tamizados se transfirieron a vasos precipitados y se introdujeron a un horno de secado Merment modelo 30-750 a $90{ }^{\circ} \mathrm{C}$, durante 24 horas o hasta que las muestras estuvieran secas. Posteriormente, se realizaron oxidaciones con el propósito de eliminar el contenido de materia orgánica presente en las muestras y reducir la interferencia en la identificación de los MP; para ello, se adicionaron de 20 a $30 \mathrm{~mL}$ de hipoclorito de sodio al $10 \%$ a cada muestra y se dejó reposar durante 24 a 48 h. Después de observar una disminución considerable de la materia orgánica, se procedió a retirar el hipoclorito, lavando los MP a possible MP concentration gradient across the bay. Also, sampling during the dry, transition and rainy seasons allows the effect that changes in precipitation and runoff have on MP concentrations to be evaluated.

Surface water samples were collected at both points (IE and EE) for each season (dry, transition and rainy), using a 250 -micron horizontal trawl with a $29.5 \mathrm{~cm}$ mouth diameter. The trawls were towed over a distance of $600.4 \pm 50.7 \mathrm{~m}$ (mean $\pm \mathrm{SD})$, for 10 minutes, at a constant speed. Each of the collected samples were replicated three times making a total of 36 samples. These samples were transferred to clean containers and subsequently refrigerated at a temperature of $4{ }^{\circ} \mathrm{C}$.

\section{Laboratory phase}

Methodologies for PM analysis proposed by Masura et al. (2015) and Invemar (2017a) were adapted for the treatment of the samples. First, each sample collected underwent a separation process, in which they were transferred with the help of distilled water to a tower of stacked stainless-steel sieves with apertures of $5 \mathrm{~mm}$ and $0.25 \mathrm{~mm}$ in diameter. The elements collected on the $5 \mathrm{~mm}$ sieve and those that passed through the $0.25 \mathrm{~mm}$ sieve were discarded, since they did not fall within the size of material to be studied. The sieved solids were transferred to beakers and placed in a Merment drying oven model $30-750$ at $90{ }^{\circ} \mathrm{C}$ for 24 hours or until the samples were dry. Subsequently, the material was oxidized in order to eliminate any organic matter present in the samples and reduce any interference they might present in the identification of the MP; for this, 20 to $30 \mathrm{~mL}$ of $10 \%$ sodium hypochlorite were added to each sample and they were left to stand for 24 to $48 \mathrm{~h}$. After a significant decrease in organic matter was observed, the hypochlorite was removed by washing the MP with distilled water in a $0.25 \mathrm{~mm}$ sieve. The washed solids were then transferred to a density separator, which contained a $5 \mathrm{M}$ saline solution, and the mixture was left to stand for one hour. Once the solids were separated by density, those that had settled were drained from the separator and inspected to ensure they contained no MP. Finally, all floating and suspended solids were collected in a Petri dish and subsequently heated in the oven at $80{ }^{\circ} \mathrm{C}$ until the samples were dry. Once dry, the samples were first inspected under a stereoscope to eliminate elements identified as not being MP due to their morphology. Finally, all MP were classified according to size and shape using a ZEISS AxioCam microscope. 
en un tamiz de $0,25 \mathrm{~mm}$ con agua destilada, los sólidos lavados se transfirieron a un separador de densidad, el cual contenía solución salina $5 \mathrm{M}$ y se dejó reposar la mezcla durante una hora. Una vez que los sólidos fueron separaron por densidades, se drenaron del separador aquellos que sedimentaron y se inspeccionaron con el fin de asegurar la inexistencia de MP. Por último, se recolectaron en una caja Petri todos los sólidos flotantes y suspendidos y posteriormente se secaron en el horno a $80{ }^{\circ} \mathrm{C}$ hasta que las muestras estuvieran secas. Una vez que los ejemplares estuvieron secos, se inspeccionaron inicialmente mediante un estereoscopio, para depurar aquellos que no se consideraban como MP según su morfología. Por último, todos los MP se clasificaron según su tamaño y forma mediante un microscopio ZEISS AxioCam.

\section{Análisis estadístico}

Para identificar la dinámica de la densidad de los diferentes tipos MP hallados (fragmentos, fibras y pellets) en los años estudiados (2015-2019), las diferentes épocas del año (seca, lluvia y transición) y las zonas de muestreo (estuario interno y externo), se desarrolló un Escalamiento Multidimensional No Métrico (nMDS), basado en matrices de similaridad a partir de distancias euclidianas y datos sin transformar (Clarke, 1993; Clarke et al., 2014). Así mismo, se evaluaron posibles diferencias en los grupos de datos mediante un Análisis de Varianza Multivariado Permutacional (Permanova), en donde se calcularon los valores de significación [p(PERM)] a partir de 9999 permutaciones, tomando $\alpha<0,05$ como diferencias estadísticamente significativas. Además, se realizaron pruebas pos hoc, Permanova pair-wise test, para identificar grupos significativamente diferentes (Clarke, 1993; Clarke et al., 2014; Anderson, 2017), también basadas en Distancias Euclidianas y datos sin transformar. Por último, se realizó un análisis de porcentaje de similitud (SIMPER), con distancia euclidiana a dos vías para identificar los tipos de MP como fuentes de similaridad entre las variables estudiadas (años, épocas y ubicación) (Nie et al., 2019; Ribeiro et al., 2019).

\section{RESULTADOS}

Los MP encontrados en los años analizados (20152019), en las diferentes épocas y puntos del estuario, correspondieron a fragmentos, fibras y pellets (Figura 2). El total promedio hallado fue 382 017,4 \pm 59 973,3 partículas $/ \mathrm{km}^{2}$ (promedio \pm error estándar), en donde la mayor cantidad correspondió a fragmentos con $237584,7 \pm 49757,5$ partículas $/ \mathrm{km}^{2}$ (62,2\%), seguido de fibras con $143021,3 \pm 19804,9$ partículas $/ \mathrm{km}^{2}(37,4 \%)$ y por último pellets con $1411,4 \pm 791,2$ partículas $/ \mathrm{km}^{2}(0,4 \%)$.

\section{Statistical analysis}

A Nonmetric Multidimensional Scaling (nMDS) method was developed, based on similar matrices constructed from Euclidean distances and untransformed data (Clarke, 1993; Clarke et al., 2014), to identify the density dynamics of the different MP types found (fragments, fibers and pellets) in the years studied (2015 and 2019), in the different seasons (dry, rainy and transition) and sampling location (inner and external estuary). Possible differences in the data groups were also evaluated using a Permutational Multivariate Analysis of Variance (Permanova), where significance values $[\mathrm{p}(\mathrm{PERM})]$ were calculated from 9999 permutations, considering $\alpha<0.05$ as statistically significant differences. Additional post hoc tests, the Permanova pair-wise test, were performed to identify significantly different groups (Clarke, 1993; Clarke et al., 2014; Anderson, 2017), also using Euclidean Distances and untransformed data. Finally, a similarity percentage analysis (SIMPER) was performed using the two-way Euclidean distance formula to identify whether the studied variables (years, seasons and location) have similarities regarding MP type collected (Nie et al., 2019; Ribeiro et al., 2019).

\section{RESULTS}

The shapes of MP found in the different seasons of the years analyzed (2015 and 2019), and at different locations in the estuary, consisted of fragments, fibers, and pellets (Figure 2). The average of total MP found was $382017.4 \pm 59973.3$ particles $/ \mathrm{km}^{2}$ (average \pm standard error), with the largest proportion of this being fragments, at $237584.7 \pm 49757.5$ particles $/ \mathrm{km}^{2}(62.2 \%)$, followed by fibers at $143021.3 \pm 19804.9$ particles $/ \mathrm{km}^{2}(37.4 \%)$ and finally pellets at $1411.4 \pm 791.2$ particles $/ \mathrm{km}^{2}(0.4 \%)$. The average sizes of the MP ranged from $88.6 \mu \mathrm{m}$ to $5878.163099 \mu \mathrm{m}$, specifically, $1075.6 \pm 116 \mu \mathrm{m}$, $1741.9 \pm 215.2 \mu \mathrm{m}$ and $353.6 \pm 115 \mu \mathrm{m}$ for fragments, fibers and pellets, respectively. 


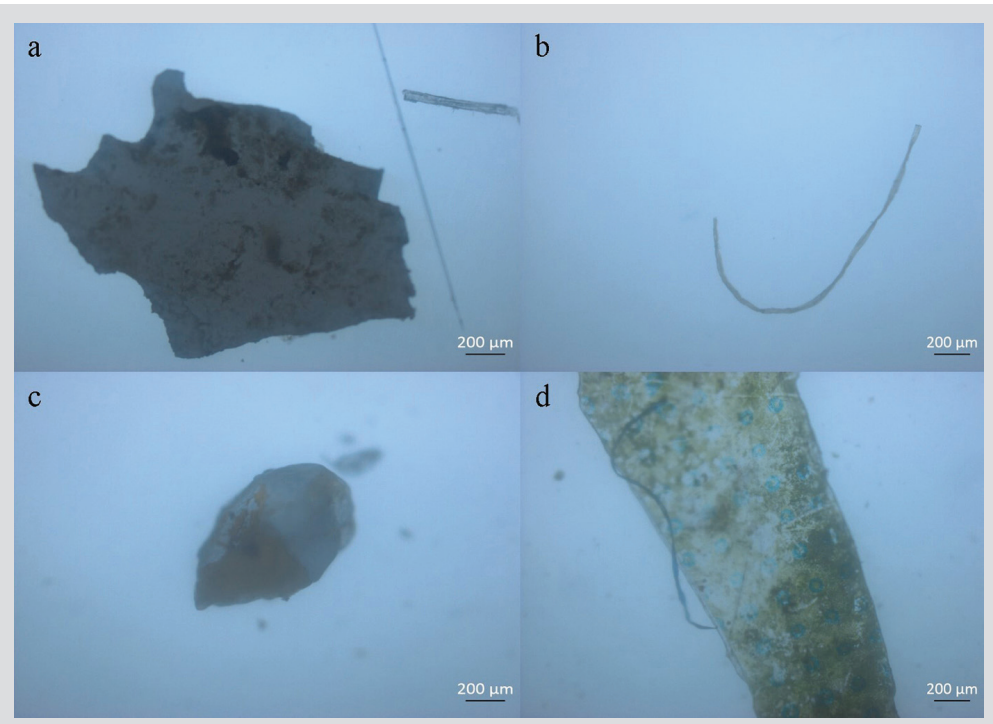

Figura 2. Tipos de microplásticos hallados en las muestras de agua superficial. (A) Fragmento, (B) Fibra, (C) Pellets, (D) Unión de fibra y fragmento.
Figure 2. Types of microplastics found in surface water samples. (A) Fragment, (B) Fiber, (C) Pellets, (D) Union of fiber and fragment.
Los MP presentaron tamaños promedios que oscilaron entre $88,6 \mu \mathrm{m}$ y $5878,163099 \mu \mathrm{m}$, específicamente, $1075,6 \pm 116 \mu \mathrm{m}, 1741,9 \pm 215,2 \mu \mathrm{m}$ y $353,6 \pm 115 \mu \mathrm{m}$ para fragmentos, fibras y pellets, respectivamente.

La abundancia de los distintos tipos de MP estudiados fue mayor en 2019 (521 $275 \pm 103671,2$ partículas $\left./ \mathrm{km}^{2}\right)$ en comparación de 2015 (242 759,8 \pm 42 203,2 partículas $\left./ \mathrm{km}^{2}\right)$, observándose una acumulación progresiva de estos elementos en la superficie del agua (Figura 3). Así mismo, se encontraron diferencias en las densidades y tamaños de los mismos entre las diferentes épocas hidroclimáticas y los sectores estudiados del estuario.
The abundance of the different types of MP studied was higher in $2019\left(521275 \pm 103671.2\right.$ particles $\left./ \mathrm{km}^{2}\right)$ compared to 2015 (242 $759.8 \pm 42203.2$ particles $\left./ \mathrm{km}^{2}\right)$, showing a progressive accumulation of these elements on the water's surface (Figure 3). Also, differences in the densities and sizes of these elements were found for the different hydroclimatic seasons and locations of the estuary chosen for the study.

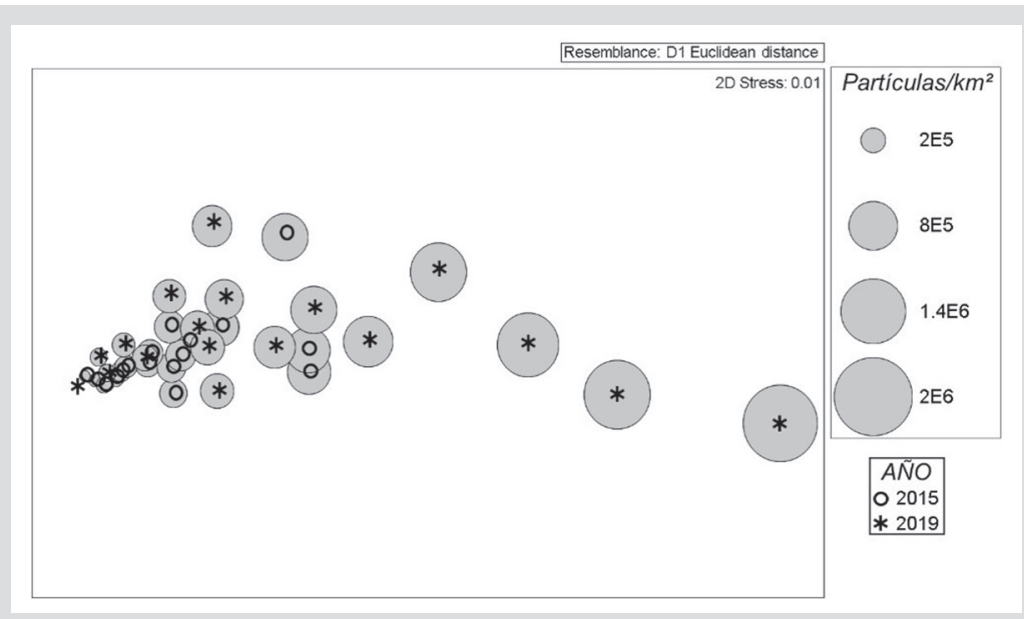

Figura 3. Densidad total de los microplásticos en 2015 y 2019.

Figure 3. Total density of microplastics in 2015 and 2019. 


\section{Microplásticos en 2015}

Para 2015 los MP que estuvieron presentes en mayor cantidad fueron los fragmentos con $143962,2 \pm 27935,8$ partículas $/ \mathrm{km}^{2}(59,3 \%$, y las fibras con $97856,7 \pm 23057$ partículas $/ \mathrm{km}^{2} \quad(40,3 \%)$; las menores cantidades las obtuvieron los pellets con 940,9 \pm 940,9 partículas $/ \mathrm{km}^{2}$ $(0,4 \%)$ (Tabla 1$)$. Los tamaños de los MP para este año variaron desde $88,6 \mu \mathrm{m}$ a $5878,2 \mu \mathrm{m}$, sus tamaños promedios fueron $1103,3 \pm 129 \mu \mathrm{m}$ para fragmentos, $1862,6 \pm 230,1 \mu \mathrm{m}$ para las fibras y $88,6 \mu \mathrm{m}$ para pellets (Tabla 2).

Tabla 1. Densidad promedio de microplásticos en 2015 y 2019 (partículas $/ \mathrm{km}^{2}$ ) (promedio \pm error estándar), en función de las épocas y zonas de muestreo. Estuario Interno (EI), Estuario Externo (EE). Los resultados de las pruebas pos hoc, permanova pair-wise test, se representan con letras $(\mathrm{p}(\mathrm{PERM})<0,05)$.

\section{Microplastics in 2015}

In 2015, MP fragments dominated the samples with $143962.2 \pm 27935.8$ particles $/ \mathrm{km}^{2}$ (59.3\%), followed by fibers with $97856.7 \pm 23057$ particles $/ \mathrm{km}^{2}(40.3 \%)$; pellets were the least common MP type with $940.9 \pm 940.9$ particles $/ \mathrm{km}^{2}(0.4 \%)$ (Table 1). MP sizes for this year ranged from $88.6 \mu \mathrm{m}$ to $5878.2 \mu \mathrm{m}$, with an average size of $1103.3 \pm 129 \mu \mathrm{m}$ for fragments, $1862.6 \pm 230.1 \mu \mathrm{m}$ for fibers and $88.6 \mu \mathrm{m}$ for pellets (Table 2).

\begin{tabular}{|c|c|c|c|c|c|c|}
\hline $\begin{array}{l}\text { Año / } \\
\text { Year }\end{array}$ & & & Total & $\begin{array}{c}\text { Fragmentos / } \\
\text { Fragments }\end{array}$ & Fibras/ Fibers & Pellets \\
\hline \multirow{12}{*}{2015} & \multirow{3}{*}{ Época / Season } & Seca / Dry & $90329.2 \pm 27542.1 \mathrm{~b}$ & $67746.9 \pm 28676$ & $22582.3 \pm 3570.6 b$ & 0 \\
\hline & & Transición / Transition & $268164.9 \pm 67664.5 \mathrm{a}$ & $177835.7 \pm 56975.6$ & $90329.2 \pm 20355.4 \mathrm{a}$ & 0 \\
\hline & & Lluvia / Rain & $369785.3 \pm 69477.3 \mathrm{a}$ & $186304.1 \pm 46280$ & $180658.5 \pm 49796.4 \mathrm{a}$ & $2822.8 \pm 2822.8$ \\
\hline & \multirow{2}{*}{ Zona / Zone } & $\mathrm{EI} / \mathrm{IE}$ & $197595.2 \pm 57643.0$ & $94093.0 \pm 16726.3$ & $101620.4 \pm 42247.6$ & $1881.9 \pm 1881.9$ \\
\hline & & $\mathrm{EE} / \mathrm{EE}$ & $287924.4 \pm 61131.5$ & $193831.5 \pm 49145.0$ & $94093 \pm 21702.7$ & 0 \\
\hline & \multirow{3}{*}{$\mathrm{EI} / \mathrm{IE}$} & Seca / Dry & $73392.5 \pm 14936.8$ & $50810.2 \pm 9778.4$ & $22582.3 \pm 5645.6$ & 0 \\
\hline & & Transición / Transition & $146785 \pm 20355.4$ & $84683.7 \pm 16936.7$ & $62101.4 \pm 5645.6$ & 0 \\
\hline & & Lluvia / Rain & $372608.1 \pm 122132.5$ & $146785 \pm 24608.5$ & $220177.5 \pm 102089.8$ & $5645.6 \pm 5645.6$ \\
\hline & \multirow{3}{*}{$\mathrm{EE} / \mathrm{EE}$} & Seca / Dry & $107266 \pm 57296.4$ & $84683.7 \pm 61066.3$ & $22582.3 \pm 5645.6$ & 0 \\
\hline & & Transición / Transition & $389544.8 \pm 88005.8$ & $270987.7 \pm 85246.3$ & $118557.1 \pm 35256.6$ & 0 \\
\hline & & Lluvia / Rain & $366962.5 \pm 95974.8$ & $225823.1 \pm 92422.1$ & $141139.4 \pm 20355.4$ & 0 \\
\hline & Total & General & $242759.8 \pm 42203.2$ & $143962.2 \pm 27935.8$ & $97856.7 \pm 23057$ & $940.9 \pm 940.9$ \\
\hline \multirow{12}{*}{2019} & \multirow{3}{*}{ Época / Season } & Seca / Dry & $231468.7 \pm 74939.6 b$ & $135493.9 \pm 61066.3 b$ & $93152 \pm 27222.0 \mathrm{~b}$ & $2822.8 \pm 2822.8$ \\
\hline & & Transición / Transition & $372608.1 \pm 99047.3 b$ & $208886.4 \pm 77078.2 b$ & $160899 \pm 34084.5 b$ & $2822.8 \pm 2822.8$ \\
\hline & & Lluvia / Rain & $959748.2 \pm 185824.4 \mathrm{a}$ & $649241.4 \pm 212021.3 \mathrm{a}$ & $310506.8 \pm 41868.7 \mathrm{a}$ & 0 \\
\hline & \multirow{2}{*}{ Zona / Zone } & $\mathrm{EI} / \mathrm{IE}$ & $346262.1 \pm 104752.1 \mathrm{~b}$ & $163721.7 \pm 80585.5 b$ & $180658.5 \pm 46124.7$ & $1881.9 \pm 1881.9$ \\
\hline & & $\mathrm{EE} / \mathrm{EE}$ & $696287.9 \pm 164460.9 \mathrm{a}$ & $498692.7 \pm 148729.4 \mathrm{a}$ & $195713.4 \pm 37988.4$ & $1881.9 \pm 1881.9$ \\
\hline & \multirow{3}{*}{$\mathrm{EI} / \mathrm{IE}$} & Seca / Dry & $186304.1 \pm 70513.2$ & $107266 \pm 90857$ & $79038.1 \pm 24608.5$ & 0 \\
\hline & & Transición / Transition & $191949.6 \pm 63622.4$ & $62101.4 \pm 20355.4$ & $124202.7 \pm 57296.4$ & $5645.6 \pm 5645.6$ \\
\hline & & Lluvia / Rain & $660532.6 \pm 220394.6$ & $321797.9 \pm 223624.7$ & $338734.6 \pm 48892.1$ & 0 \\
\hline & \multirow{3}{*}{$\mathrm{EE} / \mathrm{EE}$} & Seca / Dry & $276633.3 \pm 145147.4$ & $163721.8 \pm 97947.1$ & $107266 \pm 53855.4$ & $5645.6 \pm 5645.6$ \\
\hline & & Transición / Transition & $553266.6 \pm 111205$ & $355671.4 \pm 88005.8$ & $197595.2 \pm 34341.7$ & 0 \\
\hline & & Lluvia / Rain & $1258963.8 \pm 185876$ & $976684.9 \pm 259880.5$ & $282278.9 \pm 74684$ & 0 \\
\hline & Total & General & $521275.0 \pm 103671.2$ & $331207.2 \pm 521275$ & $188185.9 \pm 29042.7$ & $1881.9 \pm 1290.9$ \\
\hline
\end{tabular}

Table 1. Average density of microplastics in 2015 and 2019 (particles $/ \mathrm{km}^{2}$ ) (average \pm standard error), depending on the seasons and sampling location. Inner Estuary (IE), External Estuary (EE). The results of the post hoc tests, the Permanova pair-wise test, are represented by letters $(\mathrm{p}($ PERM $)<0.05)$. 
Tabla 2. Tamaños de microplásticos en 2015 y $2019(\mu \mathrm{m})$ (promedio \pm error estándar), para el total de cada año y en función de las zonas de muestreo.
Table 2. Sizes of microplastics in 2015 and $2019(\mu \mathrm{m})$ (average \pm standard error), for each year's total and for each sampling location.

\begin{tabular}{|c|c|c|c|c|c|c|}
\hline $\begin{array}{l}\text { Año / } \\
\text { Year }\end{array}$ & & Tamaño / Size $(\mu \mathrm{m})$ & $\begin{array}{l}\text { Microplásticos totales / } \\
\text { Total microplastics }\end{array}$ & $\begin{array}{c}\text { Fragmentos / } \\
\text { Fragments }\end{array}$ & Fibras / Fibers & Pellets \\
\hline \multirow{5}{*}{2015} & \multirow{3}{*}{ Total } & Promedio / Average & $1356.6 \pm 110.4$ & $1103.7 \pm 129$ & $1862.6 \pm 230.1$ & 88.6 \\
\hline & & Máximo / Maximun & 5878.2 & 5607.3 & 5878.2 & - \\
\hline & & Mínimo / Minimum & 88.6 & 232.91 & 425.3 & - \\
\hline & $\begin{array}{l}\text { Estuario interno / } \\
\text { Internal estuary }\end{array}$ & Promedio / Average & $1611.5 \pm 213.4$ & $1406.9 \pm 277$ & $1929.8 \pm 329.8$ & 88.6 \\
\hline & $\begin{array}{l}\text { Estuario externo / } \\
\text { External estuary }\end{array}$ & Promedio / Average & $1120.8 \pm 122.6$ & $911.7 \pm 107.4$ & $1748.2 \pm 286.4$ & 0 \\
\hline \multirow{5}{*}{2019} & \multirow{3}{*}{ Total } & Promedio / Average & $1048.8 \pm 258.8$ & $903.5 \pm 222.9$ & $1334.8 \pm 538$ & $486.1 \pm 139.2$ \\
\hline & & Máximo / Maximum & 4904.7 & 1898.7 & 4904.7 & 625.3 \\
\hline & & Mínimo / Minimum & 88.6 & 88.6 & 197.5 & 346.8 \\
\hline & $\begin{array}{l}\text { Estuario interno / } \\
\text { Internal estuary }\end{array}$ & Promedio / Average & $978.4 \pm 397.1$ & $685.6 \pm 56.4$ & $1341.9 \pm 896.2$ & 625.3 \\
\hline & $\begin{array}{l}\text { Estuario externo / } \\
\text { External estuary }\end{array}$ & Promedio / Average & $1898.4 \pm 267.5$ & $1266.7 \pm 589.5$ & $1323.1 \pm 156$ & 346.8 \\
\hline
\end{tabular}

La densidad de los MP tuvo diferencias significativas solo entre las épocas analizadas $(p($ PERM $)=0,012)$, en donde se presentó una mayor acumulación total en la época de lluvia (369 785,3 $\pm 69477,3$ partículas $/ \mathrm{km}^{2}$ ) y en la época de transición (268 164,9 $\pm 67664,5$ partículas $\left./ \mathrm{km}^{2}\right)$, y menor en la época seca (90 329,2 \pm 27 542,1 partículas $/ \mathrm{km}^{2}$ ) (Tabla 1). Es importante destacar que, en todas las épocas muestreadas para 2015 los fragmentos fueron el tipo de MP que predominó, respecto a los demás (Tabla 1). Por medio del Análisis Simper, se evidenció que el tipo de MP que mayor contribución tuvo para que se presentaran diferencias en la distribución fueron los fragmentos en época seca $(98,5 \%)$.

Los MP aumentaron del estuario interno hacia el estuario externo, presentándose menor cantidad estimada en el EI (197 595,2 \pm 57643 partículas $\left./ \mathrm{km}^{2}\right)$ y mayor en el EE (287 924,4 \pm 61 131,5 partículas $/ \mathrm{km}^{2}$ ) (Tabla 1). A pesar de que los fragmentos predominaron en 2015, su presencia varió en las zonas del estuario, en EI predominaron las fibras con $101620,4 \pm 42$ 247,6 partículas $/ \mathrm{km}^{2}$ (51,4\%), mientras que en EE los fragmentos con $193831,5 \pm 49145$ partículas $/ \mathrm{km}^{2}$ (67,3 \%) (Tabla 1). Por otro lado, los mayores tamaños de los MP se obtuvieron para fibras 1929,8 $\pm 329,8 \mu$ m en EI y $1748,2 \pm 286,4 \mu \mathrm{m}$ en EE (Tabla 2). El análisis Simper sugiere que la mayor contribución para las diferencias de la distribución de los MP fue en el EE para los fragmentos (92,04\%).

Para el EI, las mayores concentraciones de los MP totales se presentaron en la época lluvia, seguida por la de transición y finalmente la época seca, con
MP density only showed significant differences depending on the season analyzed $(\mathrm{p}(\mathrm{PERM})=0.012)$, with a higher total accumulation in the rainy season (369 $785.3 \pm 69477.3$ particles $/ \mathrm{km}^{2}$ ) and the transition season (268 $164.9 \pm 67664.5$ particles $\left./ \mathrm{km}^{2}\right)$, and a lower accumulation in the dry season $(90329.2 \pm 27542.1$ particles $/ \mathrm{km}^{2}$ ) (Table 1). It is important to highlight that, in all the seasons studied for 2015, fragments were the predominant type of MP compared to the others (Table 1). The Simper analysis showed that the type of MP that contributed the most to the differences between variables were fragments collected in the dry season $(98.5 \%)$.

The amount of MP increased the further from the inner estuary and closer to the external estuary the samples were taken, with the lowest estimated amount in the IE (197595.2 \pm 57643 particles $\left./ \mathrm{km}^{2}\right)$ and the highest in the EE (287 $924.4 \pm 61131.5$ particles $/ \mathrm{km}^{2}$ ) (Table 1). Although fragments predominated overall in 2015, their presence varied depending on the area of the estuary, in the IE fibers predominated with $101620.4 \pm 42247.6$ particles $/ \mathrm{km}^{2}$ (51.4\%), while in the EE fragments were the most common MP type with $193831.5 \pm 49145$ particles $/ \mathrm{km}^{2}$ (67.3\%) (Table 1). Meanwhile, fibers had the largest MP sizes at $1929.8 \pm 329.8 \mu \mathrm{m}$ in the IE and $1748.2 \pm 286.4 \mu \mathrm{m}$ in the EE (Table 2). Simper analysis suggests that the MP type that made the greatest contribution to the differences in MP distribution was fragments found in the EE $(92.04 \%)$. 
promedios de $372608,1 \pm 122132,5$ partículas $/ \mathrm{km}^{2}$, seguido $146785,0 \pm 20355,4$ partículas $/ \mathrm{km}^{2}$ y $73392,5 \pm 14936,8$ partículas $/ \mathrm{km}^{2}$, respectivamente (Tabla 1). En esta localización todos los tipos de MP se presentaron en mayor medida durante la época de lluvia. Los MP que estuvieron en mayor concentración fueron las fibras (220 177,5 \pm 102 089,8 partículas $\left./ \mathrm{km}^{2}\right)$ y los fragmentos $(146785,0 \pm 24608,5$ partículas $/ \mathrm{km}^{2}$ ), por el contrario, los pellets se hallaron en menor cantidad respecto a los demás y sólo se encontraron en la época de lluvia (Tabla 1). En cuanto al EE, la mayor cantidad de MP se presentaron durante la época de transición (389 544,8 $\pm 88005,8$ partículas $/ \mathrm{km}^{2}$ ), seguido por época de lluvia (366 962,5 $\pm 95974,8$ partículas $/ \mathrm{km}^{2}$ ) y por último época seca $\left(107266 \pm 572\right.$ 96,4 partículas $/ \mathrm{km}^{2}$ ) (Tabla 1). Los tipos de MP que predominaron fueron los fragmentos, durante la época de transición (270 987,7 $\pm 85246,3$ partículas $/ \mathrm{km}^{2}$ ) y las fibras durante la época de lluvia (141 139,4 $\pm 20355,4$ partículas $\left./ \mathrm{km}^{2}\right)$. No se encontraron pellets en ninguna época del año (Tabla 1).

Microplásticos en 2019

En cuanto a 2019 los MP que se presentaron en mayor cantidad fueron los fragmentos, con una densidad promedio de $331207,2 \pm 91558,2$ partículas $/ \mathrm{km}^{2}$ (63,5\%), posteriormente, las fibras con $188185,9 \pm 29042,7$ partículas $/ \mathrm{km}^{2}(36,1 \%)$, y finalmente los pellets con $1881,9 \pm 1290,9$ partículas $/ \mathrm{km}^{2}$ $(0,4 \%)$ (Tabla 1). Los tamaños de los MP presentes oscilaron entre $88,6 \mu \mathrm{m}$ y $4904,7 \mu \mathrm{m}$. (Tabla 2).

Por otro lado, se encontraron diferencias significativas en la cantidad de MP encontrada entre las diferentes épocas estudiadas (lluvia, transición y seca) $(\mathrm{p}(\mathrm{PERM})=0,0011)$ $y$ entre las zonas muestreadas $(\mathrm{p}(\mathrm{PERM})=0,0133)$. Para las épocas, las mayores cantidades de MP totales se registraron en el periodo de lluvia (959 748,2 $\pm 185824,4$ partículas $/ \mathrm{km}^{2}$ ) y las menores en transición y el periodo seco, con densidades promedio de $372608,1 \pm 99047,3$ partículas $/ \mathrm{km}^{2}$ y $231468,7 \pm 74939,6$ partículas $/ \mathrm{km}^{2}$, respectivamente (Tabla 1). Las mayores cantidades según el tipo de MP correspondieron a los fragmentos (649 241,4 $\pm 212021,3$ partículas $/ \mathrm{km}^{2}$ ) y las fibras (310 506,8 $\pm 41868,7$ partículas $/ \mathrm{km}^{2}$ ), en época de lluvia (Tabla 1). Por medio del análisis Simper, se evidenció que el tipo de MP que mayor contribución tuvo para que se presentaran diferencias en la distribución fueron los fragmentos en época lluvia $(93,6 \%)$.

Para 2019, al igual que para 2015, se encontró que las concentraciones de MP aumentaron a medida que la distancia a la zona urbana y de desembocadura de ríos fue mayor, esto se evidenció con las cantidades de MP halladas, en donde fue menor en el EI (346 262,1 $\pm 104752,1$ partículas $\left./ \mathrm{km}^{2}\right)$ y mayor en el
The highest concentrations of total MP in the IE occurred during the rainy season, followed by the transition and finally the dry season, with averages of $372608.1 \pm 122132.5$ particles $/ \mathrm{km}^{2}$, followed by $146785.0 \pm 20355.4$ particles $/ \mathrm{km}^{2}$ and $73392.5 \pm 14936.8$ particles $/ \mathrm{km}^{2}$, respectively (Table 1 ). At this location, there were more of all types of MP during the rainy season. The most common type of MP were fibers (220 $177.5 \pm 102089.8$ particles $\left./ \mathrm{km}^{2}\right)$ and fragments $\left(146785.0 \pm 24608.5\right.$ particles $\left./ \mathrm{km}^{2}\right)$, whereas pellets were found in lower quantities compared to the others and were only found in the rainy season (Table 1). In the EE, the largest amount of MP was found during the transition period (389 $544.8 \pm 88005.8$ particles $/ \mathrm{km}^{2}$ ), followed by the rainy season (366 $962.5 \pm 95974.8$ particles $/ \mathrm{km}^{2}$ ) and the least amount in the dry season $\left(107266 \pm 57296.4\right.$ particles $\left./ \mathrm{km}^{2}\right)$ (Table 1). The most common MP types during the transition period were fragments $\left(270987.7 \pm 85246.3\right.$ particles $\left./ \mathrm{km}^{2}\right)$ and during the rainy season more fibers were collected (141 139.4 \pm 20355.4 particles $/ \mathrm{km}^{2}$ ). No pellets were found at any time of year (Table 1).

\section{Microplastics in 2019}

In 2019, the most common type of MP were fragments, with an average density of $331207.2 \pm 91558.2$ particles $/ \mathrm{km}^{2}$ (63.5 \%), followed by fibers with $188185.9 \pm 29042.7$ particles $/ \mathrm{km}^{2}$ (36.1\%), and finally pellets with $1881.9 \pm 1290.9$ particles $/ \mathrm{km}^{2}(0.4 \%)$ (Table 1). The sizes of the MP ranged from $88.6 \mu \mathrm{m}$ to $4904.7 \mu \mathrm{m}$. (Table 2).

There were significant differences in the amount of MP found in each of the different seasons studied (rainy, transition and dry $(\mathrm{p}(\mathrm{PERM})=0.0011)$ and between the locations sampled $(\mathrm{p}(\mathrm{PERM})=0.0133)$. For the seasons, the highest amounts of total MP were recorded in the rainy season (959 748.2 \pm 185824.4 particles $/ \mathrm{km}^{2}$ ) and the lowest in the transition and dry seasons, with average densities of $372608.1 \pm 99047.3$ particles $/ \mathrm{km}^{2}$ and $231468.7 \pm 74939.6$ particles $/ \mathrm{km}^{2}$, respectively (Table 1 ). The most common MP type were fragments (649 $241.4 \pm 212021.3$ particles $/ \mathrm{km}^{2}$ ) and fibers (310 506.8 \pm 41868.7 particles $/ \mathrm{km}^{2}$ ), in the rainy season (Table 1). The Simper analysis showed that the type of MP that contributed the most to the differences in distribution were fragments collected in the rainy season (93.6\%).

In 2019, as in 2015, MP concentrations increased the further the sample was taken from urban areas and river mouths, evidenced by smaller quantities of MP found in the 
EE (696 287,9 \pm 164 460,9 partículas/km²) (Tabla 1). En el EI predominaron las fibras con $180658,5 \pm 46124,7$ partículas $/ \mathrm{km}^{2}$ (52,2 \%) y en EE los fragmentos con 498 692,7 \pm 148 729,4 partículas $/ \mathrm{km}^{2}$ (71,6\%) (Tabla 1). Los mayores tamaños de MP encontrados, para ambas zonas, fueron los de las fibras (Tabla 2). El análisis Simper sugiere que la mayor contribución para las diferencias de la distribución de los MP fue en el EI por los fragmentos $(90,3 \%)$.

También se observaron diferencias significativas de la concentración de los MP en la interacción de zonas y épocas de muestreo. Se encontró que para elEI las mayores concentraciones totales de MP se presentaron en época de lluvia, seguida por la época de transición (660 532,6 $\pm 220394,6$ partículas $/ \mathrm{km}^{2}$, $191949,6 \pm 63622,4$ partículas $/ \mathrm{km}^{2}$, respectivamente) y que en la época seca se presentaron un menor número de partículas (186 304,1 \pm 70 513,2 partículas $/ \mathrm{km}^{2}$ ) (Tabla 1). Además, se encontró que las fibras se presentaron con mayor concentración respecto a los demás MP (338 734,6 448 892,1 partículas $/ \mathrm{km}^{2}$ ) en época de lluvia, seguido por los fragmentos en esta misma época (321 797,9 $\pm 223624,7$ partículas $\left./ \mathrm{km}^{2}\right)$. Los pellets sólo se presentaron en época de transición para el EI $\left(5645,6 \pm 5645,6\right.$ partículas $\left./ \mathrm{km}^{2}\right)$ (Tabla 1). Para el EE, al igual que el EI; la época de mayor concentración de MP fue la de lluvia $1258963,8 \pm 185876$ partículas $/ \mathrm{km}^{2}$, seguida de transición con $553266,6 \pm 111205$ partículas $/ \mathrm{km}^{2}$ y la época seca 276 633,3 \pm 145 147,4 partículas $/ \mathrm{km}^{2}$ (Tabla 1). Los MP que se presentaron en mayores concentraciones fueron los fragmentos y las fibras durante la época de lluvia (976 684,9 $\pm 259880,5$ partículas $/ \mathrm{km}^{2}, 282$ 278,9 \pm 74684 partículas $/ \mathrm{km}^{2}$, respectivamente) (Tabla 1).

Aumento en la contaminación por

microplásticos de 2015 a 2019

Teniendo en cuanta ambos años, se encontró que para 2019 hubo un aumento 114,7 \% de la densidad total de los MP con relación al 2015, pasando de $242759,8 \pm 42$ 203,2 partículas $/ \mathrm{km}^{2}$ en 2015 a $521275 \pm 103671,2$ partículas $/ \mathrm{km}^{2}$ en 2019, sugiriendo una tasa de aumento promedio anual del 28,7 \%. Así mismo, se observó que para los tres tipos de MP analizados hubo un incremento de sus cantidades de 2015 a 2019 (Figura 4a). Adicionalmente, se encontraron diferencias en las densidades de los MP entre épocas y zonas del estuario. Para los dos años estudiados, las mayores concentraciones de MP se observaron en las épocas de lluvia (664 766,7 \pm 129 828,1 partículas $\left./ \mathrm{km}^{2}\right)$ y transición $(320 \quad 386,5 \quad \pm \quad 59 \quad 313,7$ partículas $/ \mathrm{km}^{2}$ ), mientras que en la época seca se presentaron en menores cantidades (160 $899 \pm 43606$ partículas $/ \mathrm{km}^{2}$ ) (Figura $4 \mathrm{~b})$. La concentración de los MP aumentó en ambos años al
IE $\left(346262.1 \pm 104752.1\right.$ particles $\left./ \mathrm{km}^{2}\right)$ and larger quantities in the EE (696 $287.9 \pm 164460.9$ particles $\left./ \mathrm{km}^{2}\right)$ (Table 1). In the IE, fibers were the most common type of MP with $180658.5 \pm 46124.7$ particles $/ \mathrm{km}^{2}(52.2 \%)$ and in the EE, fragments were most common with $498692.7 \pm 148729.4$ particles $/ \mathrm{km}^{2}$ (71.6 \%) (Table 1). In both zones, fibers were found to have the largest MP sizes (Table 2). The Simper analysis suggests that the MP type with the greatest contribution to the differences in the distribution of MPs was fragments collected in the IE (90.3\%).

There were also significant differences in the concentration of MP between the zones and seasons sampled. The highest total concentrations of MP were found in the rainy season, followed by the transition season (660 532.6 \pm 220394.6 particles $/ \mathrm{km}^{2}, 191949.6 \pm 63622.4$ particles $/ \mathrm{km}^{2}$, respectively) and the lowest number of particles in the dry season $\left(186304.1 \pm 70513.2\right.$ particles $\left./ \mathrm{km}^{2}\right)$ (Table 1). Also, there was a higher concentration of fibers than other MP (338 $734.6 \pm 48892.1$ particles $\left./ \mathrm{km}^{2}\right)$ in the rainy season, followed by fragments in this same season (321 $797.9 \pm 223624.7$ particles $/ \mathrm{km}^{2}$ ). In the IE, pellets were only found during the transition season $(5645.6 \pm 5645.6$ particles $/ \mathrm{km}^{2}$ )(Table 1). For the EE, similar to the IE, the season with the highest concentration of MP was the rainy season with $1258963.8 \pm 185876$ particles $/ \mathrm{km}^{2}$, followed by the transition with $553266.6 \pm 111205$ particles $/ \mathrm{km}^{2}$ and the dry season with $276633.3 \pm 145147.4$ particles $/ \mathrm{km}^{2}$ (Table 1). The highest concentrations of MPs were found to be fragments and fibers during the rainy season $(976684.9 \pm 259880.5$ particles $/ \mathrm{km}^{2}, \quad 282278.9 \pm 74684$ particles $/ \mathrm{km}^{2}$, respectively) (Table 1 ).

Increase in microplastic pollution from 2015 to 2019

The results for both years show an increase of $114.7 \%$ in total MP density in 2019 compared to 2015, from $242759.8 \pm 42203.2$ particles $/ \mathrm{km}^{2}$ in 2015 to $521275 \pm 103671.2$ particles $/ \mathrm{km}^{2}$ in 2019 , indicating an average annual rate of increase of $28.7 \%$. Similarly, it was observed that for the three types of MP analyzed there was an increase in each from 2015 to 2019 (Figure 4a). Additionally, differences in MP densities were found depending on the season and estuary zone. For both years studied, the highest MP concentrations were observed in the rainy $\left(664766.7 \pm 129828.1\right.$ particles $\left./ \mathrm{km}^{2}\right)$ and transition seasons $\left(320386.5 \pm 59313.7\right.$ particles $/ \mathrm{km}^{2}$ ), while in the dry season they occurred in lower amounts (160 $899 \pm 43606$ particles $/ \mathrm{km}^{2}$ ) (Figure 4b). In both years, concentrations of MP increased the further away from the 
incrementarse la distancia desde la zona urbana y de la zona de ingreso de los ríos hacia al mar, observándose una menor densidad de MP en el EI (271 928,7 $\pm 60734,9$ partículas $\left./ \mathrm{km}^{2}\right)$ y una mayor densidad de MP en el EE (492 106,2 \pm 98467,1 partículas $/ \mathrm{km}^{2}$ ) (Figura 4c). En el EI, las fibras predominaron durante la época de lluvia (279 456,1 $\pm 57143,2$ partículas $\left./ \mathrm{km}^{2}\right)$ y transición (93 $152 \pm 29253,7$ partículas $/ \mathrm{km}^{2}$ ), respecto a los demásMP, mientasenelEElos fragmentos predominarondurante las épocas lluvia, transición y seca $(211709,2 \pm 208340.2$ partículas $/ \mathrm{km}^{2}, 158076,2 \pm 57973,8$ partículas $/ \mathrm{km}^{2}$ y 64 924,1 \pm 54 560,9 partículas $/ \mathrm{km}^{2}$, respectivamente). En el EI se presentó una mayor cantidad de MP totales durante la época de lluvia (516 570,3 $\pm 129780,8$ partículas $/ \mathrm{km}^{2}$ ), seguido por la época de transición (169 367,3 $\pm 31534,5$ partículas $/ \mathrm{km}^{2}$ ) y seca (129 848,3 \pm 40945 partículas $/ \mathrm{km}^{2}$ ), del mismo modo ocurrió en el EE con promedios de $812963,1 \pm 220307,8$ partículas $/ \mathrm{km}^{2}, \quad 471 \quad 405,7 \pm 73 \quad 229,5$ partículas $/ \mathrm{km}^{2}$, 191 949,6 479 400,2 partículas/ $/ \mathrm{km}^{2}$ para las épocas lluvia, transición y seca, respectivamente. De manera general, los MP que predominaron en el estudio fueron los fragmentos $(62,2 \%)$, seguido de las fibras (37,4\%). Cabe destacar que se presentó un aumento significativo de los fragmentos hallados en el estuario desde 2015 a 2019 (130,1 \%) (Figura 4a). urban area and the river inlets and the closer to the sea the samples were taken, with a lower density of MP in the IE (271 $928.7 \pm 60734.9$ particles $/ \mathrm{km}^{2}$ ) and a higher density of MP in the EE (492 106.2 \pm 98467.1 particles $/ \mathrm{km}^{2}$ ) (Figure $4 c)$. In the IE, the most common type of MP were fibers during the rainy season $\left(279456.1 \pm 57143.2\right.$ particles $\left./ \mathrm{km}^{2}\right)$ and the transition (93 $152 \pm 29253.7$ particles $/ \mathrm{km}^{2}$ ), while in the EE, fragments were most common during the rainy, transition and dry seasons $(211709.2 \pm 208340.2$ particles $/ \mathrm{km}^{2}, 158076.2 \pm 57973.8$ particles $/ \mathrm{km}^{2}$ and $64924.1 \pm 54560.9$ particles $/ \mathrm{km}^{2}$, respectively). In the IE, the highest amount of total MP was recorded during the rainy season $\left(516570.3 \pm 129780.8\right.$ particles $\left./ \mathrm{km}^{2}\right)$, followed by the transition $\left(169367.3 \pm 31534.5\right.$ particles $\left./ \mathrm{km}^{2}\right)$ and dry seasons (129 $848.3 \pm 40945$ particles $/ \mathrm{km}^{2}$ ), The same occurred in the EE with averages of $812963.1 \pm 220307.8$ particles $/ \mathrm{km}^{2}, \quad 471 \quad 405.7 \pm 73229.5$ particles $/ \mathrm{km}^{2}$, $191949.6 \pm 79400.2$ particles $/ \mathrm{km}^{2}$ for the rainy, transition and dry seasons, respectively. Overall, MP fragments dominated the study $(62.2 \%)$, followed by fibers (37.4\%). It should be noted that there was a significant increase in fragments found in the estuary from 2015 to 2019 (130.1\%) (Figure 4a).

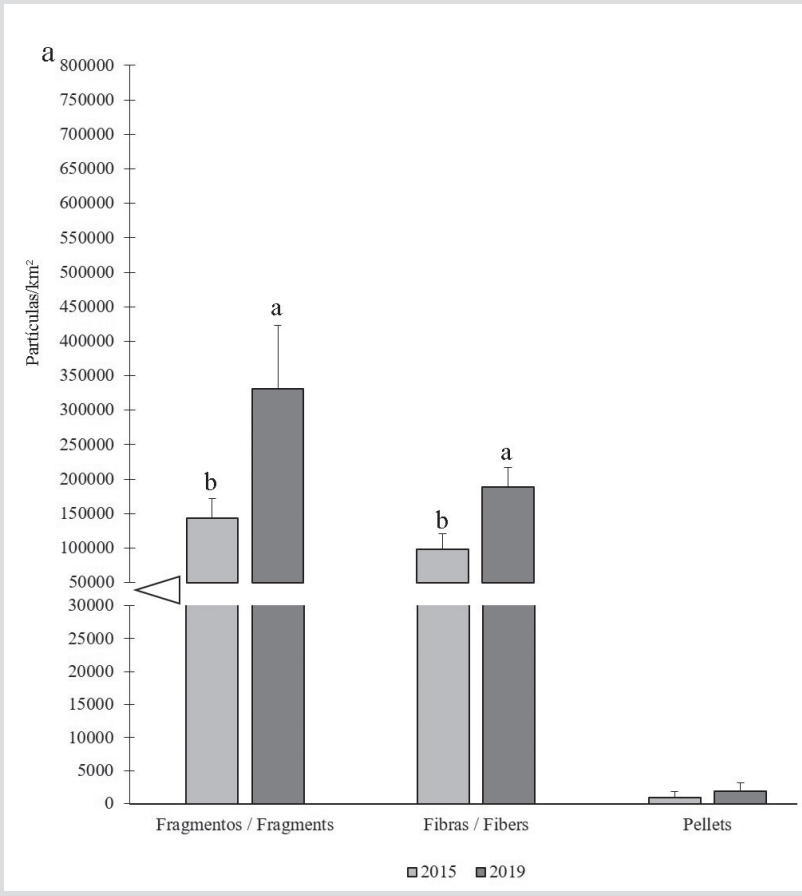

Figura 4. a) Densidad promedio de los tipos de microplásticos para 2015 (gris claro) y 2019 (gris oscuro). b) Densidad promedio del total de los microplásticos para las épocas analizadas. c) Densidad promedio del total de los microplásticos para los sectores analizados. Los resultados de las pruebas pos hoc, permanova pair-wise test, se representan con letras $(\mathrm{p}(\mathrm{PERM})<0,05)$.

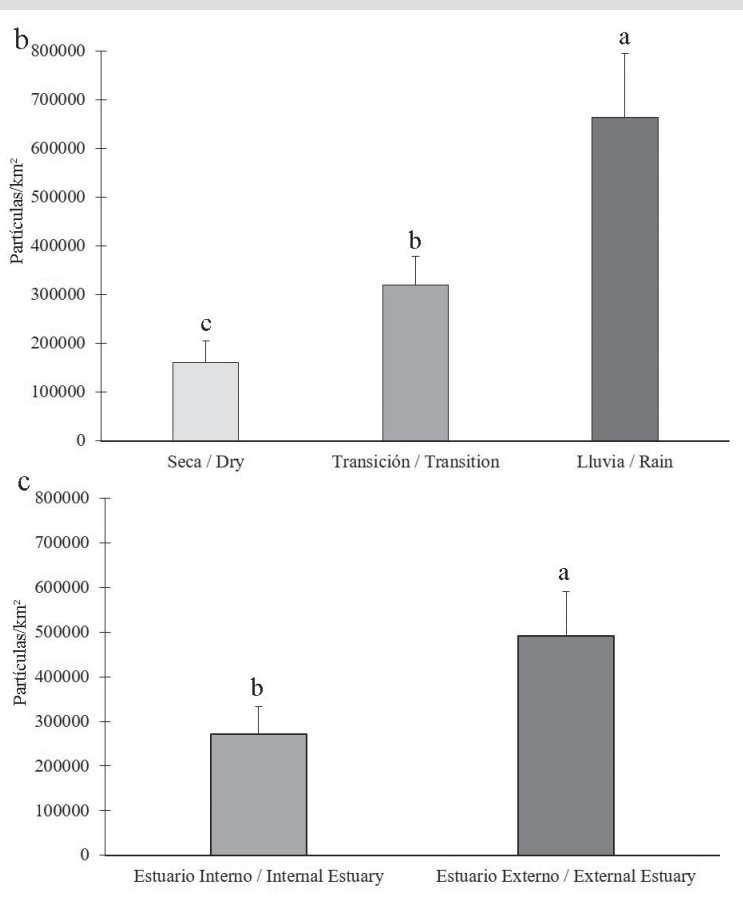

Figure 4. a) Average density of the types of microplastics for 2015 (light gray) and 2019 (dark gray). b) Average density of the total microplastics for the seasons analyzed. c) Average density of all microplastics for the locations analyzed. The results of the post hoc tests, the Permanova pairwise test, are represented by letters $(\mathrm{p}(\mathrm{PERM})<0.05)$. 
Tabla 3. Densidad de microplásticos presentes en cuerpos de aguas naturales superficiales de diferentes partes del mundo (promedios \pm desviación estándar). Los valores mencionados en el apartado a, fueron convertidos en potencias de 10 a la 6 para fines comparativos.
Table 3. Density of microplastics in natural bodies of surface water in different parts of the world (average \pm standard deviation). The values included in section a, were converted into powers of 10 to 6 for comparative purposes.

\begin{tabular}{|c|c|c|c|c|}
\hline & Localización del estudio/ Study location & $\begin{array}{l}\text { Año / } \\
\text { Year }\end{array}$ & $\begin{array}{c}\text { Densidad (Partículas } / \mathbf{k m}^{2} \text { ) / } \\
\text { Density (Particles } / \mathbf{k m}^{2} \text { ) }\end{array}$ & Referencia/ Reference \\
\hline \multirow{10}{*}{ 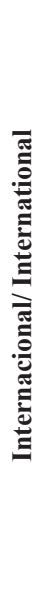 } & $\begin{array}{l}\text { Sistemas de estuarios de Solent, Reino Unido / } \\
\text { Solent estuarine system, UK }\end{array}$ & 2018 & $5.00 \times 10^{6}$ & Anderson et al. (2018) \\
\hline & Bahía de Tokio, Japón / Tokyo Bay, Japan & 2017 & $3.20 \times 10^{6}$ & Matsuguma et al. (2017) \\
\hline & Bahía Skenderun, Turquía / Skenderun Bay, Turkey & 2017 & $1.10 \times 10^{6}$ & Gündoğdu (2017) \\
\hline & Bahía de Buenaventura, Colombia / Buenaventura Bay, Colombia & 2019 & $0.50 \times 10^{6}$ & Este estudio \\
\hline & Bahía de Hiroshima, Japón / Hiroshima Bay, Japan & 2018 & $0.20 \times 10^{6}$ & Sagawa et al. (2018) \\
\hline & Océano Atlántico / Atlantic Ocean & 2014 & $0.10 \times 10^{6}$ & Eriksen et al. (2014) \\
\hline & Océano Pacífico noroccidental / Northwest Pacific Ocean & 2014 & $0.10 \times 10^{6}$ & Eriksen et al. (2014) \\
\hline & Mar Mediterráneo noroccidental / Northwest Mediterranean Sea & 2018 & $0.10 \times 10^{6}$ & Schmidt et al. (2018) \\
\hline & Isla de Pascua, Pacífico sur / Easter Island, South Pacific & 2017 & $0.06 \times 10^{6}$ & Eriksen et al. (2014) \\
\hline & Bahía de Bengala, India / Bay of Bengal, India & 2014 & $0.02 \times 10^{6}$ & Ory et al. (2017) \\
\hline \multirow{9}{*}{$\frac{\frac{\pi}{0}}{\frac{\hat{\theta}}{\Xi}}$} & $\begin{array}{l}\text { Bahía de Buenaventura, Valle del Cauca / } \\
\text { Buenaventura Bay, Valle del Cauca }\end{array}$ & 2019 & $2.80 \pm 1.90$ & Este estudio \\
\hline & Bahía de Cartagena y Barú, Bolívar / Cartagena Bay and Barú, Bolívar & 2017 & $2.46 \pm 3.87$ & Invemar y MADS (2017) \\
\hline & $\begin{array}{l}\text { Bahía de Buenaventura y bahía Málaga, Valle del Cauca / } \\
\text { Buenaventura and Málaga Bays, Valle del Cauca }\end{array}$ & & $0.73 \pm 0.93$ & \\
\hline & Capurganá y Sapzurro, Chocó / Capurganá and Sapzurro, Chocó & & $0.49 \pm 0.58$ & \\
\hline & Bahía de Tumaco, Nariño / Tumaco Bay, Nariño & & $0.47 \pm 0.58$ & \\
\hline & Golfo de Urabá, Antioquia / Gulf of Urabá, Antioquia & & $0.37 \pm 0.30$ & \\
\hline & $\begin{array}{l}\text { Bahía de Santa Marta, El Rodadero y Taganga, Magdalena / } \\
\text { Bay of Santa Marta, El Rodadero and Taganga, Magdalena }\end{array}$ & & $0.29 \pm 0.27$ & \\
\hline & $\begin{array}{l}\text { Spratt Bight y Jhonny Cay, San Andrés Isla / } \\
\text { Spratt Bight and Jhonny Cay, San Andrés Island }\end{array}$ & & $0.15 \pm 0.23$ & \\
\hline & $\begin{array}{l}\text { Bahía Solano y Golfo de Tribugá, Chocó / } \\
\text { Bahía Solano and Gulf of Tribugá, Chocó }\end{array}$ & & $0.11 \pm 0.09$ & \\
\hline
\end{tabular}

Al comparar las concentraciones de los MP en aguas superficiales del estuario de la bahía de Buenaventura $\left(0,5 \times 10^{6}\right.$ partículas $\left./ \mathrm{km}^{2}\right)$, con las concentraciones registradas en aguas superficiales de otras regiones costeras, se podría sugerir que Buenaventura tiene un nivel de contaminación intermedio, con tendencia a alto (Tabla 3), especialmente si se tiene en cuenta la tasa de aumento en la concentración de microplásticos promedio anual fue de $28,7 \%$. Por otra parte, a nivel local, al comparar las concentraciones de los MP en aguas superficiales del estuario de la bahía de Buenaventura $\left(2,8\right.$ partículas $\left./ \mathrm{m}^{3}\right)$ con las concentraciones encontradas en diferentes ambientes marino-costeros de Colombia, se encontró que la bahía de Buenaventura presenta una mayor cantidad de microplásticos en el agua, que los registrados para otras áreas costeras de Colombia.
Comparing the concentrations of MP in surface waters of the Buenaventura Bay estuary $\left(0.5 \times 10^{6}\right.$ particles $/ \mathrm{km}^{2}$ ) with the concentrations recorded in surface waters of other coastal regions suggests that Buenaventura has an intermediate level of contamination, with a tendency to high (Table 3), especially if the rate of increase of the average annual concentration of microplastics is considered, which is $28.7 \%$. On the other hand, at the local level, when comparing the concentrations of MP in surface waters of the Buenaventura Bay estuary $\left(2.8\right.$ particles $\left./ \mathrm{m}^{3}\right)$ with the concentrations found in different marine-coastal environments in Colombia, it was found that Buenaventura Bay has a higher amount of microplastics in the water than those recorded for other coastal areas of Colombia. 


\section{DISCUSIÓN}

La cantidad de MP presentes en agua superficial de la bahía de Buenaventura se incrementó un 114,7\% desde 2015 a 2019; este incremento es un posible resultado de distintas características que posee el lugar de estudio y sus alrededores. Buenaventura es una de las ciudades portuarias más reconocidas de Sudamérica, la cual duplicó la población en los últimos 30 años, alcanzando a albergar 423,927 personas (Ávila Arias y Toro Cárdenas, 2003). Sólo estas características, sumando al ineficiente manejo de residuos sólidos y a la poca o ausente cultura de cuidado ambiental que poseen sus habitantes (Suárez Manzano y Suárez Montaño, 2015), hacen que las cantidades de residuos depositadas a los cuerpos de agua que confluyen en los estuarios, sean cada vez mayores, explicando el gran aumento de los MP encontrados en la bahía.

También es importante considerar que el puerto de Buenaventura, por su cercanía a la línea del ecuador, es cercano a las principales rutas marítimas que atraviesan el planeta, lo cual le permite ser un puerto concentrador y de transbordo (Sociedad Portuaria Regional de Buenaventura S.A, 2017). Debido a esto, sus aguas, y por ende las de la bahía, podrían estar expuestas a la contaminación por MP, generada por los buques de tránsito internacional, a través de las aguas de lastre. A pesar de que el agua de lastre optimiza la seguridad y estabilidad de las embarcaciones, es una importante fuente de contaminación. Cuando se aprovecha el agua del medio marino del puerto en que se encuentre la embarcación y luego se descargan en el puerto de destino, se liberan en éste de forma involuntaria diferentes elementos y organismos que no son propios del ecosistema (GarcíaMendoza, 2015).

Los fragmentos fueron el tipo de MP que se presentó con mayor concentración durante 2015 y 2019 (59,3\% y 63,5\% del total de los MP, respectivamente). Esta dinámica coincide con un estudio realizado en el golfo Pérsico (Kor y Mehdinia, 2020), en donde se encontró que los fragmentos predominaron en dicha investigación con $41 \%$ del total de MP. Por otro lado, en el océano Pacífico noroccidental, los fragmentos representaron $80 \%$ de los MP totales analizados (Pan et al., 2019). Así mismo, en Colombia, en un estudio realizado en el manglar de la Ciénaga Grande de Santa Marta los fragmentos fueron uno de los MP que predominaron (Invemar y MinAmbiente, 2018), del mismo modo, los fragmentos predominaron en investigaciones realizados en los departamentos de Nariño,

\section{DISCUSSION}

The amount of MP in the surface waters of Buenaventura Bay increased by $114.7 \%$ from 2015 to 2019; this increase possibly results from the characteristics of the study site and its surroundings. Buenaventura is one of the most well-known port cities in South America, and its population has doubled in the last 30 years, and is now home to 423,927 people (Ávila Arias and Toro Cárdenas, 2003). These factors alone, together with the inefficient management of solid waste and its inhabitants' limited or lack of culture of environmental protection (Suárez Manzano and Suárez Montaño, 2015), mean that the amount of waste deposited in the bodies of water that flow into the estuaries is increasing, explaining the large increase in the amount of MP found in the bay.

It is also important to consider that the port of Buenaventura's proximity to the equator means that the world's principal shipping routes run close by, and it is therefore a hub and transshipment port (Sociedad Portuaria Regional de Buenaventura S.A., 2017). Owing to this, its waters, and those of the bay, could be exposed to MP pollution generated by the ballast water of international transit vessels. Ballast water is used to improve the safety and stability of ships, but it is an also a major source of pollution. Vessels take on sea water from their port of origin and then discharge it at the destination port, unintentionally releasing different substances and organisms that do not belong to that ecosystem (García-Mendoza, 2015).

Fragments were the type of MP that occurred with the highest concentration during 2015 and 2019 (59.3\% and $63.5 \%$ of total MP, respectively). This is in line with a study carried out in the Persian Gulf (Kor and Mehdinia, 2020), where fragments were found to predominate, representing $41 \%$ of total MP. In the northwestern Pacific Ocean, too, fragments accounted for $80 \%$ of the total MP analyzed (Pan et al., 2019). Likewise, in Colombia, in a study conducted in the mangrove swamp of the Ciénaga Grande de Santa Marta, fragments were one of the most common MP recovered (Invemar and MinAmbiente, 2018), and similarly, fragments predominated in research conducted in the departments of Nariño, Valle del Cauca, Bolívar and Chocó (Caribbean) (42\%, 69\%, $83 \%$ and $58 \%$, respectively) (Invemar and MADS, 2017).

Due to the irregular shape of these MP, it can be inferred that they are secondary MP, i.e., they are derived from the fragmentation of larger pieces. A possible reason 
Valle del Cauca, Bolívar y Chocó (Caribe) (42\%, $69 \%$, $83 \%$ y $58 \%$, respectivamente) (Invemar y MADS, 2017).

Debido a la forma irregular que presentaron estos MP se puede inferir que son secundarios, es decir, que provienen de la fragmentación de elementos de mayor tamaño. Una posible razón de la gran presencia de estos MP en la superficie es su menor densidad en comparación a la del agua estuarina, por lo cual no sólo los fragmentos, sino también las fibras (tipo de MP que también presentan altas concentraciones en este estudio), por su amplia superficie y su pequeña masa, flotan y se acumulan rápidamente en la superficie (Pan et al., 2019). Esto también puede explicar la abundancia de los MP en EE en los dos años estudiados; zona del estuario que tiene una mayor densidad al presentar una fuerte influencia marina, lo cual favorece que los MP se presenten en mayor medida sobre la superficie del agua y no en el fondo.

La precipitación tuvo una clara influencia en la cantidad de MP. En la época de lluvia, la escorrentía y el caudal de los ríos aumenta (Támalo et al., 2016), finalizando su recorrido en el océano Pacífico. En el municipio de Buenaventura se evidencian prácticas en donde el manejo de los residuos sólidos es inadecuado, no solo por la ausencia de educación ambiental, sino también porque carecen de sistemas de correcta disposición de residuos (Suárez Manzano y Suárez Montaño, 2015), presentándose un aporte directo y mezcla continua de los cuerpos de agua dulce que transportan materiales contaminantes y llegan a los estuarios. Adicionalmente, la región no cuenta con un sistema adecuado de tratamiento de aguas residuales, lo cual aumenta el riesgo de contaminación. Debido a esto, por la acción de la escorrentía los MP son transportados hasta el océano, proceso que se ve intensificado con un incremento de las lluvias en la zona del estuario y en la cuenca de los ríos afluentes.

Por último, el considerable aumento de los MP en el estuario de la bahía de Buenaventura sugiere que se tomen medidas pertinentes para que se evite un continuo incremento de las concentraciones a través de tiempo y se contrarresten los posibles efectos nocivos sobre la vida marina y en la salud humana. Los MP, específicamente los fragmentos, representan una grave amenaza a nivel ecológico. En primer lugar, por su pequeño tamaño, lo cual hace que muchos organismos los ingieran de forma más fácil, aumentando su biodisponibilidad a través de las redes tróficas y, en segundo lugar, porque los fragmentos concentran y magnifican los contaminantes orgánicos for the large amount of these MP on the surface is that they have a lower density than the estuary water. Fragments, as well as fibers (a MP type that this study found in high concentrations), have large surface areas and small mass, and so float and accumulate quickly on the surface (Pan et al., 2019). This may also explain the abundance of MP in the EE in the two years studied; this part of the estuary has a higher density due to its proximity to the open sea, resulting in a greater presence of MP on the surface of the water rather than on the bottom.

Precipitation had a clear influence on the amount of MP collected. During the rainy season, runoff and river flows increase (Támalo et al., 2016), ending their journey in the Pacific Ocean. In the municipality of Buenaventura, it has been shown that much of the solid waste management is inadequate, not only due to the absence of environmental education, but also because of the lack of proper waste disposal systems (Suárez Manzano and Suárez Montaño, 2015), resulting in a direct contribution to and continuous mixing of freshwater bodies that carry polluting materials to the estuaries. Furthermore, the region does not have an adequate wastewater treatment system, which increases the risk of contamination. As a result, MP are washed into the ocean by runoff, a process that is intensified by increased rainfall in the estuary zone and in the tributary river basins.

Finally, the considerable increase of MP in the Buenaventura Bay estuary prompts the adoption of corresponding measures to avoid a continuous increase in levels of MP over time and to counteract the possible harmful effects on marine life and human health. MP, specifically MP fragments, represent a serious ecological threat. First, this is due to their small size, which makes them more easily ingested by many organisms, meaning their bioavailability through the food webs increases, and second, because fragments concentrate and magnify persistent organic pollutants in water (Saeed et al., 2020), affecting various organisms. A greater effort to study and protect estuarine ecosystems is needed not only in Buenaventura Bay, but also around the world, since it is through these ecosystems that most MP enter the marine environment (Zhang et al., 2019). Furthermore, estuaries provide environmental services such as the regulation of imbalances and disturbances, nutrient recycling, habitats, and shelter for various organisms (many of commercial interest), not to mention the recreational, aesthetic and educational services they offer to human populations. 
persistentes en el agua (Saeed et al., 2020), afectando a diversos organismos. Estos ecosistemas necesitan ser estudiados y preservados con mayor detalle no sólo en la bahía de Buenaventura, sino también alrededor de todo el mundo, ya que a través de éstos ingresan la mayoría de MP al medio marino (Zhang et al., 2019); además, los estuarios brindan servicios ambientales como regulación de desequilibrios y perturbaciones, reciclaje de nutrientes, es hábitat y refugio de diversos organismos (muchos son de interés comercial), sin mencionar los servicios de recreación, estéticos y de educación que ofrecen a las poblaciones humanas.

\section{CONCLUSIONES}

En el periodo de estudio, la concentración de MP creció $114,7 \%$ de 2015 a 2019 , que representa en promedio un aumento de $28,7 \%$ anual en la densidad de MP en las aguas superficiales de la bahía de Buenaventura. Así mismo, se evidenció un aumento en la densidad de MP hacia la zona externa de la bahía, debido probablemente al gradiente de densidad (debido a la salinidad), que permite que las partículas, en especial los fragmentos, floten por más tiempo y se puedan desplazar mayores distancias en el estuario externo. El incremento de la escorrentía, debido al aumento de las precipitaciones, contribuyó al aumento de la concentración de MP en la época de lluvia. El incremento de los microplásticos confirma la necesidad de profundizar en el estudio de la problemática de contaminación por plásticos en la bahía de Buenaventura. La presencia de los microplásticos en los ecosistemas estuarinos representa un riesgo para el ecosistema y los servicios ecosistémicos derivados.

\section{AGRADECIMIENTOS}

Los autores agradecen a la Universidad Nacional de Colombia por el apoyo institucional y económico mediante el proyecto "Efectos de operaciones de dragado en la comunidad de organismos estuarinos de la bahía de Buenaventura, Pacífico colombiano" (código 34779) y el proyecto "Efectos de los cambios en la calidad del agua en las comunidades de macroinvertebrados y peces del estuario bahía de Buenaventura" (código 42118). Así mismo, se agradece al grupo de investigación en Ecología y Contaminación Acuática por el apoyo en el campo y laboratorio.

\section{CONCLUSIONS}

In the timeframe of the study, from 2015 to 2019 , the concentration of MP grew $114.7 \%$, which represents a $28.7 \%$ annual increase on average in the density of MP on the surface waters of Buenaventura Bay. There was also evidence of an increase in MP density towards the outer zone of the bay, probably due to the density gradient (due to salinity), which allows particles, especially fragments, to float longer and travel greater distances in the external estuary. Increased runoff, due to increased rainfall, contributed to the increase in MP concentration in the rainy season. The increase in microplastics confirms the need to further study the problem of plastic pollution in Buenaventura Bay. The presence of microplastics in estuarine ecosystems represents a risk to the ecosystem itself and to the ecosystem services derived from it.

\section{ACKNOWLEDGEMENTS}

The authors would like to thank Universidad Nacional de Colombia for its institutional and financial support through the project "Effects of dredging operations on estuarine organism communities in Buenaventura Bay, Colombian Pacific" (code 34779) and the project "Effects of changes in water quality on macroinvertebrate and fish communities in the Buenaventura Bay estuary" (code 42118). We also wish to thank the Aquatic Ecology and Contamination research group for their support both in the field and in the laboratory. 


\section{BIBLIOGRAFÍA / LITERATURE CITED}

Alimba, C.G. and C. Faggio. 2019. Microplastics in the marine environment: Current trends in environmental pollution and mechanisms of toxicological profile. Environ. Toxicol. Pharmacol., 68: 61-74. https://doi.org/10.1016/j.etap.2019.03.001

Anderson, M.J. 2017. Permutational Multivariate Analysis of Variance (PERMANOVA): 1-15. In: Wiley StatsRef: Statistics Reference Online. Wiley, Chichester, UK. https://doi.org/10.1002/9781118445112.stat07841

Anderson, Z.T., A.B. Cundy, I.W. Croudace, P.E. Warwick, O. Celis-Hernández and J.L. Stead. 2018. A rapid method for assessing the accumulation of microplastics in the sea surface microlayer (SML) of estuarine systems. Sci. Rep., 8: 9428. https://doi.org/10.1038/s41598-018-27612-w

Ávila Arias, S.E. y J.C. Toro Cárdenas. 2003. Entre lo vernáculo y lo contemporáneo reinterpretación de la vivienda palafítica en Buenaventura. Degree project, Univ. Nal. Col., Manizales.

Botterell, Z.L., N. Beaumont, T. Dorrington, M. Steinke, R.C. Thompson and P.K. Lindeque. 2019. Bioavailability and effects of microplastics on marine zooplankton: A review. Environ. Pollut., 245: 98-110. https://doi.org/10.1016/j.envpol.2018.10.065

Cantera, J.R. and J.F. Blanco. 2001. The estuary ecosystem of Buenaventura Bay, Colombia: 265-280. In: Seeliger, U. and B. Kjerfve (Eds.) Coastal marine ecosystems of Latin America.

Ecosystems of Latin America. Springer, Berlin, Heidelberg. https://doi.org/10.1007/978-3-662-04482-7_19

Carbery, M., W.O. Connor and P. Thavamani. 2019. Trophic transfer of microplastics and mixed contaminants in the marine food web and implications for human health. Environ. Int., 115: 400-409. https://doi.org/10.1016/j.envint.2018.03.007

Caron, A., C. Thomas, K. Berry, C. Motti, E. Ariel and J. Brodie. 2018. Ingestion of microplastic debris by green sea turtles (Chelonia mydas) in the Great

Barrier Reef: Validation of a sequential extraction protocol. Mar. Pollut. Bull., 127: 734-751. https://doi.org/10.1016/j.marpolbul.2017.12.062

Caruso, G. 2019. Microplastics as vectors of contaminants. Mar. Pollut. Bull., 146: 921-924. https://doi.org/10.1016/j.marpolbul.2019.07.052

Chan H.S.H. , C. Dingle and C. Not. 2019. Evidence for non-selective ingestion of microplastic in demersal fish. Mar. Pollut. Bull.,149: 110523. https://doi. org/10.1016/j.marpolbul.2019.110523

Clarke, K.R. 1993. Non-parametric multivariate analyses of changes in community structure. Austral. Ecol., 18: 117-143. https://doi. org/10.1111/j.1442-9993.1993.tb00438.x

Clarke, K.R., R.N. Gorley, P.J. Somerfield and R.M. Warwick. 2014. Change in marine communities: an approach to statistical analysis and interpretation. 3rd ed. PRIMER-E Ltd., Plymouth, UK.

Cole, M., P. Lindeque, C. Halsband and T.S. Galloway. 2011. Microplastics as contaminants in the marine environment: a review. Mar. Pollut. Bull., 62:2588-2597. https://doi.org/10.1016/j.marpolbul.2011.09.025

Das, S., S. Lee, P. Kumar and K. Kim. 2019. Solid waste management: Scope and the challenge of sustainability. J. Clean. Prod., 228: 658-678. https://doi. org/10.1016/j.jclepro.2019.04.323

Devasahayam, S., G.B. Raju and C.M. Hussain. 2019. Utilization and recycling of end of life plastics for sustainable and clean industrial processes including the iron and steel industry. Mater. Sci. Energy Technol., 2: 634-646. https://doi.org/10.1016/j.mset.2019.08.002 .

Díaz, J.M. 2007. Deltas y estuarios de Colombia. Imprelibro, Cali.

Duque, G., D.E. Gamboa-García, A. Molina and P. Cogua. 2020. Effect of water quality variation on fish assemblages in an anthropogenically impacted tropical estuary, Colombian Pacific. Environ. Sci. Pollut. Res., 27: 25740-25753. https://doi.org/10.1007/s11356-020-08971-2

Eriksen, M., L.C.M. Lebreton, H.S. Carson, M. Thiel, C.J. Moore, J.C. Borerro, F. Galgani, P.G. Ryan and J. Reisser. 2014. Plastic pollution in the world's oceans: more than 5 trillion plastic pieces weighing over 250,000 tons afloat at sea. PLoS One., 9: 1-15. https://doi.org/10.1371/journal.pone.0111913

García, D.E.G., G. Duque y P. Cogua. 2018. Dinámica de la estructura y composición de macroinvertebrados y su relación con las variables ambientales en la bahía de Buenaventura. Bol. Invest. Mar. Cost., 47: 67-83. https://doi.org/10.25268/bimc.invemar.2018.47.1.738

Garcia Mendoza, R.E. 2015. Regulación para el control y prevención de la contaminación marina ocasionada por las aguas de lastre en el puerto marítimo del distrito de Cartagena. Degree project, Univ. Cart.

Giacomo, C., S. Gorbi, M. Milan, M. Benedetti, D. Fattorini, M. Pauletto, L. Bargelloni and F. Regoli. 2015. Pollutants bioavailability and toxicological risk from microplastics to marine mussels. Environ. Pollut., 198: 211-222. https://doi.org/10.1016/j.envpol.2014.12.021

Gündoğdu, S. 2017. High level of micro-plastic pollution in the Iskenderun Bay NE Levantine coast of Turkey. Aegean J. Fish. Aquat. Sci., 34: 401-408. https://doi.org/10.12714/egejfas.2017.34.4.06

Guzmán, D., J. Ruíz y M. Cadena. 2014. Regionalización de Colombia según la estacionalidad de la precipitación media mensual, a través análisis de componentes principales (ACP). IDEAM, $55 \mathrm{p}$.

Guzzetti, E., A. Sureda, S. Tejada and C. Faggio. 2018. Microplastic in marine organism: environmental and toxicological effects. Environ. Toxicol. Pharmacol., 64: 164-171. https://doi.org/10.1016/j.etap.2018.10.009

Invemar. 2005. Informe del estado de los ambientes marinos y costeros en Colombia: Año 2005. Serie Publ. Per. Invemar, 8, 360 p.

Invemar. 2018. Diagnóstico y evaluación de la calidad de las aguas marinas y costeras en el Caribe y Pacífico colombianos. Bayona-Arenas, M. y O. GarcésOrdóñez. (Eds.) Red de vigilancia para la conservación y protección de las aguas marinas y costeras de Colombia - Redcam: Invemar, MinAmbiente, Coralina, Corpoguajira, Corpamag, CRA, Cardique, Carsucre, CVS, Corpourabá, Codechocó, CVC, CRC y Corponariño. Inf. Técn. Serie Publ. Per. Invemar, 4, 336 p. 
Invemar y MinAmbiente. 2018. Cuarto Informe técnico. Convenio Interadministrativo 659 de 2017. Santa Marta, Colombia. 374 p.

Invemar y MADS. 2017. Resolución No. 646 de 2017 Formulación de lineamientos, medidas de conservación, manejo y uso de ecosistemas marinos y costeros, con la intención de apoyar acciones de fortalecimiento en la gestión ambiental de las zonas costeras de Colombia. Invemar, Santa Marta. $278 \mathrm{p}$.

Invemar. 2017a. Informe del estado de los ambientes y recursos marinos y costeros en Colombia, 2016. Serie Publ. Per., 3, 200 p.

Invemar. 2017b. Protocolo de muestreo y análisis de microplásticos en aguas marinas superficiales, sedimentos de playas y tracto digestivo de peces. Componente 5: Diagnóstico de microplásticos en zonas costeras de Colombia, Resolución 646 MinAmbiente. Invemar, Santa Marta. 21 p.

Invemar y CVC. 2015. Proyecto determinación de objetivos de calidad por usos del recurso hídrico en la bahía de Buenaventura - sector Cascajal, a través de la modelación de coliformes y dbo para definir diferentes escenarios de calidad del agua. Invemar, Santa Marta. $116 \mathrm{p}$.

Kor, K. and A. Mehdinia. 2020. Neustonic microplastic pollution in the Persian Gulf. Mar. Pollut. Bull., 150: 1-19. https://doi.org/10.1016/j. marpolbul.2019.110665

Lima, A.R.A., M. Barletta, M.F. Costa, J.A.A. Ramos, D.V. Dantas, P.A.M.C. Melo, A.K.S. Justino and G.V.B. Ferreira. 2016. Changes in the composition of ichthyoplankton assemblage and plastic debris in mangrove creeks relative to moon phases. J. Fish Biol., 89: 619-640. https://doi.org/10.1111/ jfb. 12838

Ma, B., W. Xue, Y. Ding and C. Hu. 2019. Removal characteristics of microplastics by Fe-based coagulants during drinking water treatment. J. Environ. Sci., 78: 267-275. https://doi.org/10.1016/j.jes.2018.10.006

Martínez, O., A.E. Molina y G. Duque. 2019. Influencia de la materia orgánica en la abundancia de poliquetos (Annelida: Polychaeta) de la bahía de Buenaventura, Pacífico colombiano. In: Campos Campos, N.H. y A. Acero P. (Eds.) Ciencias del mar - Una mirada desde la Universidad Nacional de Colombia. Univ. Nal. Col., Bogotá. 228 p.

Masura, J., J. Baker, G. Foster, C. Arthur and C. Herring. 2015. Laboratory methods for the analysis of microplastics in the marine environment: recommendations for quantifying synthetic particles in waters and sediments. Natl. Ocean. Atmos. Adm., 18 p.

Matsuguma, Y., H. Takada, H. Kumata, H. Kanke, S. Sakurai, T. Suzuki, M. Itoh, Y. Okazaki, B. Ruchaya, M. Pauzi Zakaria and S. Weerts. 2017. Microplastics in sediment cores from Asia and Africa as indicators of temporal trends in plastic pollution. Arch. Environ. Contam. Toxicol., 73: 230-239. https:// doi. org/10.1007/s00244-017-0414-9

Mohsen, M., Q. Wang, I. Zhang, L. Sun, C. Lin and H. Yang. 2019. Microplastic ingestion by the farmed sea cucumber Apostichopus japonicus in China. Environ. Pollut., 245: 1071-1078.

Molina, A., G. Duque and P. Cogua. 2020. Influences of environmental conditions in the fish assemblage structure of a tropical estuary. Mar. Biodivers., 50: 5. https://doi.org/10.1007/s12526-019-01023-0

Nie, H., J. Wang, X. Kaihang, Y. Huang and M. Yan. 2019. Microplastic pollution in water and fish samples around Nanxun Reef in Nansha. Sci. Total Environ., 696: 1-16. https://doi.org/https://doi.org/10.1016/j.scitotenv.2019.134022

Ory, N., P. Sobral, J., Ferreira and M. Thiel. 2017. Amberstripe scad Decapterus muroadsi (Carangidae) fish ingest blue microplastics resembling their copepod prey along the coast of Rapa Nui (Easter Island) in the South Pacific subtropical gyre. Sci. Total Environ., 586: 430-437. https://doi. org/10.1016/j.scitotenv.2017.01.175

Otero, L.J. 2005. Aplicación de un modelo hidrodinámico bidimensional para describir las corrientes y la propagación de la onda de marea en la bahía de Buenaventura. Bol. Cient. CCCP, 9-21. https://doi.org/10.26640/01213423.12.9

Pan, Z., Q. Liu, Y. Sun, X. Sun and H. Lin. 2019. Environmental implications of microplastic pollution in the northwestern Pacific Ocean. Mar. Pollut. Bull., 146: 215-224. https://doi.org/10.1016/j.marpolbul.2019.06.031

Provencher, J.F., J.C. Vermaire, S. Avery-Gomm, B.M. Braune and M.L. Mallory. 2018. Garbage in guano? Microplastic debris found in faecal precursors of seabirds known to ingest plastics. Sci. Total Environ., 664: 1477-1484. https://doi.org/10.1016/j.scitotenv.2018.07.101

Ribeiro, I.E., L. Neves dos Santos and A. Filippo González. 2019. Effects of temperature and salinity on bilateral symmetry of Eugerres brasilianus (Cuvier, 1830) larvae: Implications for Brazilian mojarra rearing. Aquaculture., 512: 734327. https://doi.org/10.1016/j.aquaculture.2019.734327

Saeed, T., N. Al-Jandal, A. Al-Mutairi and H. Taqi. 2020. Microplastics in Kuwait marine environment: Results of first survey. Mar. Pollut. Bull., 152: 110880. https://doi.org/10.1016/j.marpolbul.2019.110880

Sagawa, N., K. Kawaai and H. Hinata. 2018. Abundance and size of microplastics in a coastal sea: Comparison among bottom sediment, beach sediment, and surface water. Mar. Pollut. Bull., 133: 532-542. https://doi.org/10.1016/j.marpolbul.2018.05.036

Sarma, V.V. and H. Delabehera. 2019. New and primary production in the western Indian Ocean during fall monsoon. Mar. Chem., 215: 1-22. https://doi. org/10.1016/j.marchem.2019.103687

Schmidt, N., D. Thibault, F., Galgani, A., Paluselli and R. Sempéré. 2018. Occurrence of microplastics in surface waters of the Gulf of Lion (NW Mediterranean Sea). Prog. Oceanogr., 163: 214-220. https://doi.org/10.1016/j.pocean.2017.11.010

Singh, B. and N. Sharma . 2008. Mechanistic implications of plastic degradation. Polym. Degrad. Stab., 93: 561-584. https://doi.org/10.1016/j. polymdegradstab.2007.11.008

Sociedad Portuaria Regional de Buenaventura S.A. 2017. Ubicación geográfica. URL http://www.sprbun.com/web/portal/ubicacion-del-puerto

Suárez Manzano, H.G. y G. Suárez Montaño, G. 2015. Propuesta para la elaboración de una política pública para el manejo y aprovechamiento de los residuos sólidos en el distrito de Buenaventura Valle del Cauca. Degree project, Univ. Icesi., Cali. 
Tafurt, D., Molina, A., and Duque, G. (2021). Hábitos alimentarios de tres especies de peces lenguados (Pleuronectiformes : Achiridae) en la Bahía de Buenaventura, Pacífico Colombiano. Revista de Biología Tropical, 69, 1055-1068. https://doi.org/10.15517/rbt.v69i3.41922

Támalo, A., R. Bermudez, L. Garibaldi y A. Chavez. 2016. Erosión y escorrentía en respuesta a lluvias simuladas e incendios en bosques secos de montaña. Cienc. Suelo, 34, 105-111.

Troncoso, W., L. Vivas, L. Narváez, J. Sánchez, S. Narváez, L. Echeverry y J. Parra. 2009. Diagnóstico y evaluación de la calidad ambiental marina en el Caribe y Pacífico colombiano. Red de vigilancia para la conservación y protección de las aguas marinas y costeras de Colombia-REDCAM. Inf. Técn. Invemar, Santa Marta. $185 \mathrm{p}$.

Vanapalli, K.R., B.K. Dubey and J. Bhattacharya. 2019. Emissions and environmental burdens associated with plastic solid waste management: 313-342. In: Plastics to Energy. Elsevier. https://doi.org/10.1016/B978-0-12-813140-4.00012-1

Vásquez, D., A. Molina and G. Duque. 2021. Spatial distribution and increase of microplastics over time in sediments of Buenaventura Bay, Colombian Pacific. Bol. Invest. Mar. Cost., 50: 27-42. https://doi.org/10.25268/bimc.invemar.2021.50.1

Zhang, J., C. Zhang, Y. Deng, R. Wang, J. Wang, J. Bai, J. Wu and Y. Zhou. 2019. Microplastics in the surface water of small-scale estuaries in Shanghai. Mar. Pollut. Bull., 149, 110569. https://doi.org/10.1016/j.marpolbul.2019.110569

Zhao, S., T. Wang, L. Zhu, P. Xu, X. Wang, L., Gao and D. Li. 2019. Analysis of suspended microplastics in the Changjiang estuary: Implications for riverine plastic load to the ocean. Water Res., 161: 560-569. https://doi.org/10.1016/j.watres.2019.06.019

Zhu, J., X. Yu, Q. Zhang, Y. Li, S. Tan, D. Li, Z. Yang and J. Wang. 2019. Cetaceans and microplastics: First report of microplastic ingestion by a coastal delphinid, Sousa chinensis. Sci. Total Environ., 659: 649-654. https://doi.org/10.1016/j.scitotenv.2018.12.389 